\title{
Halogen and sulfur oxidation of germanium and tin dications
}

Riccardo Suter, ${ }^{\mathrm{a},{ }^{*}}$ Ala'aeddeen Swidan, ${ }^{\mathrm{b}}$ Charles L. B. Macdonald, ${ }^{\mathrm{b}}$ Neil Burford,,${ }^{\mathrm{a},}$, Michael J. Ferguson $^{\mathrm{c}}$

${ }^{a}$ Department of Chemistry, University of Victoria, Victoria, British Columbia V8W 3V6, Canada.

${ }^{b}$ Department of Chemistry and Biochemistry, University of Windsor, Windsor, Ontario N9B 3P4, Canada

${ }^{c}$ X-ray Crystallography Laboratory, Department of Chemistry, University of Alberta, Edmonton, Alberta T6G 2G2, Canada

\section{Supporting Information}

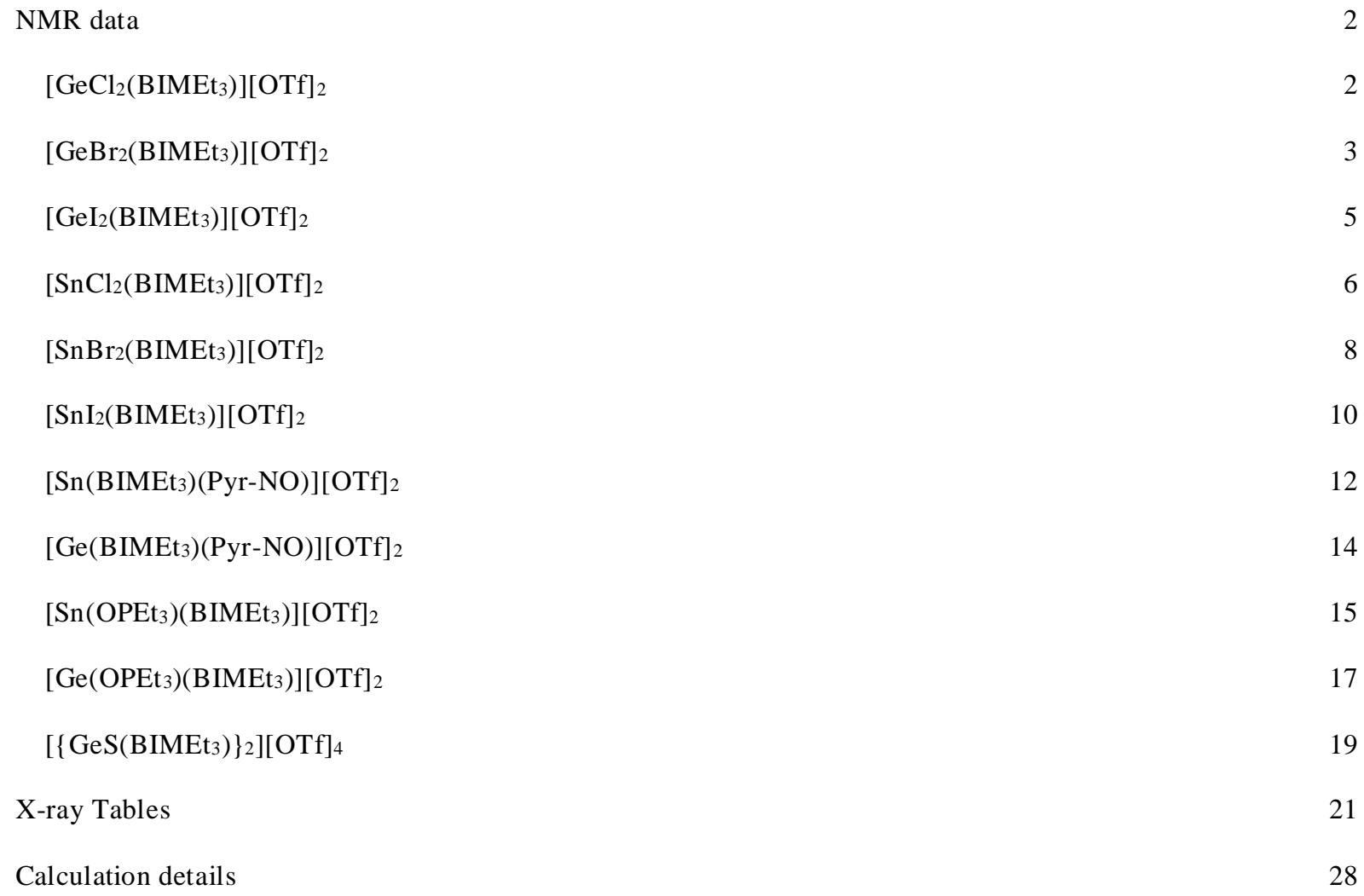




\section{NMR data}

NMR signals belon ging to volatile solvents such as diethyl ether and $\mathrm{CH}_{2} \mathrm{Cl}_{2}$ are present as inpurities in the deuterated solvent coming from the glove box atmosphere.

\section{$\left[\mathrm{GeCl}_{2}\left(\mathrm{BIMEt}_{3}\right)\right][\mathrm{OTf}]_{2}$}

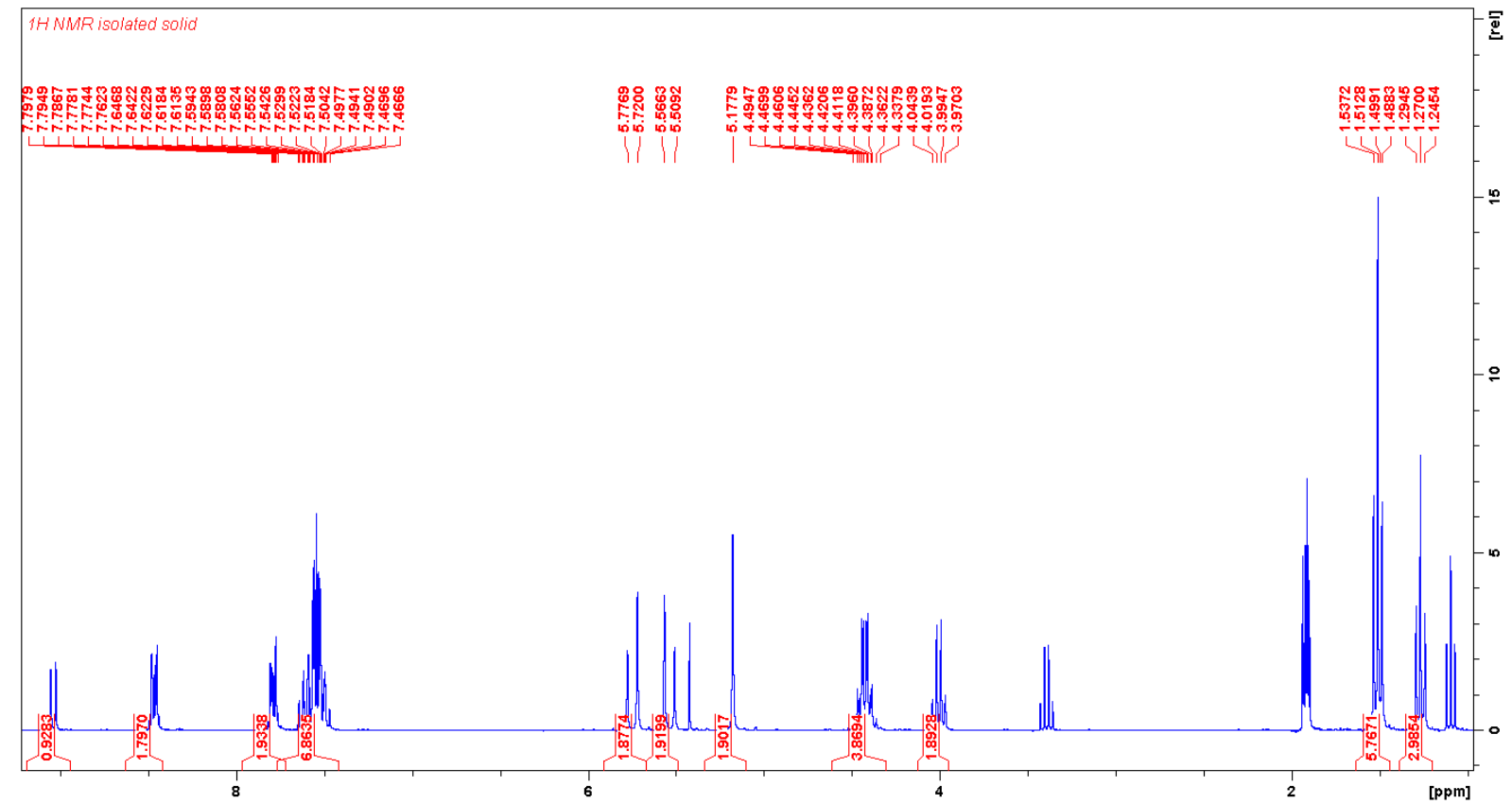

Scheme S1: ${ }^{1} \mathrm{H}$ NMR spectrum of $\left[\mathrm{GeCl}_{2}\right.$ (BIMEt 3$\left.)\right][\mathrm{OTf}]_{2}$, with signals belonging to diethyl ether (3.4 and 1.1 $\mathrm{ppm})$ and for $\mathrm{CH}_{2} \mathrm{Cl}_{2}(5.44 \mathrm{ppm})$.

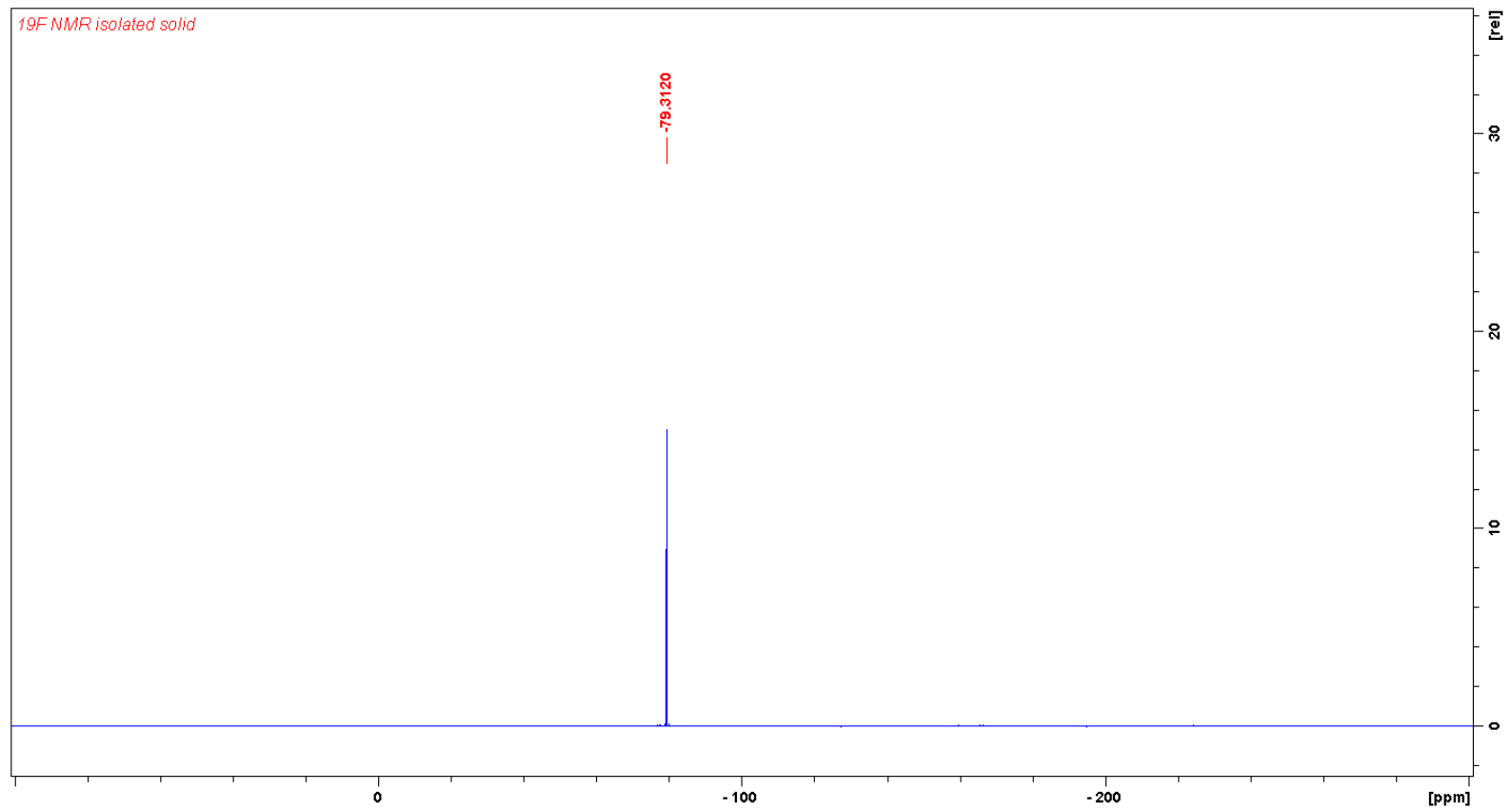

Scheme S2: ${ }^{19} \mathrm{~F}$ NMR spectrum of $\left[\mathrm{GeCl}_{2}\left(\mathrm{BIMEt}_{3}\right)\right][\mathrm{OTf}]_{2}$ 


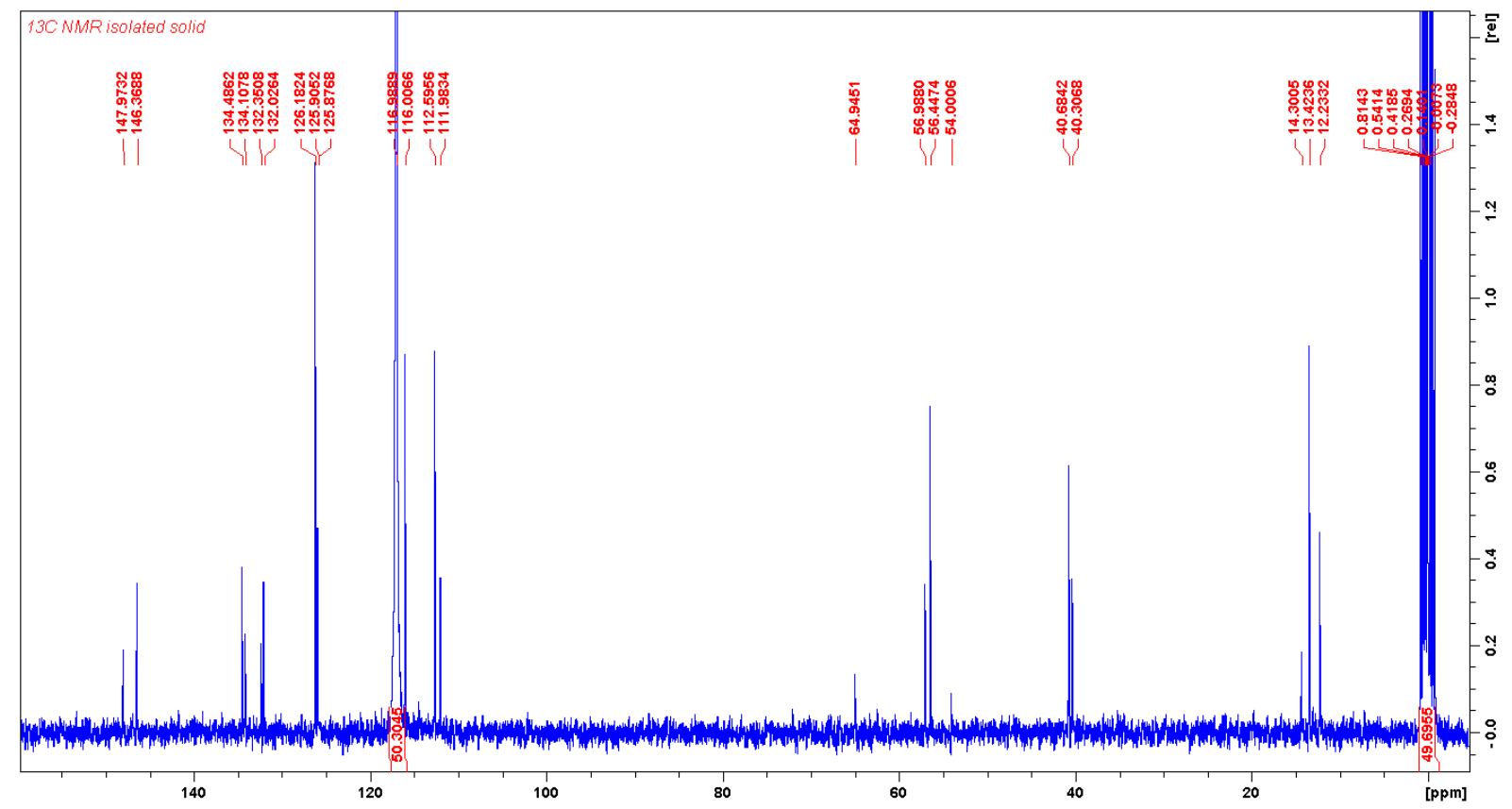

Scheme S3: ${ }^{13} \mathrm{C}$ NMR spectrum of $\left[\mathrm{GeCl}_{2}\left(\mathrm{BIMEt}_{3}\right)\right][\mathrm{OTf}]_{2}$

$\left[\operatorname{GeBr}_{2}\left(\right.\right.$ BIMEt$\left.\left._{3}\right)\right][\text { OTf }]_{2}$

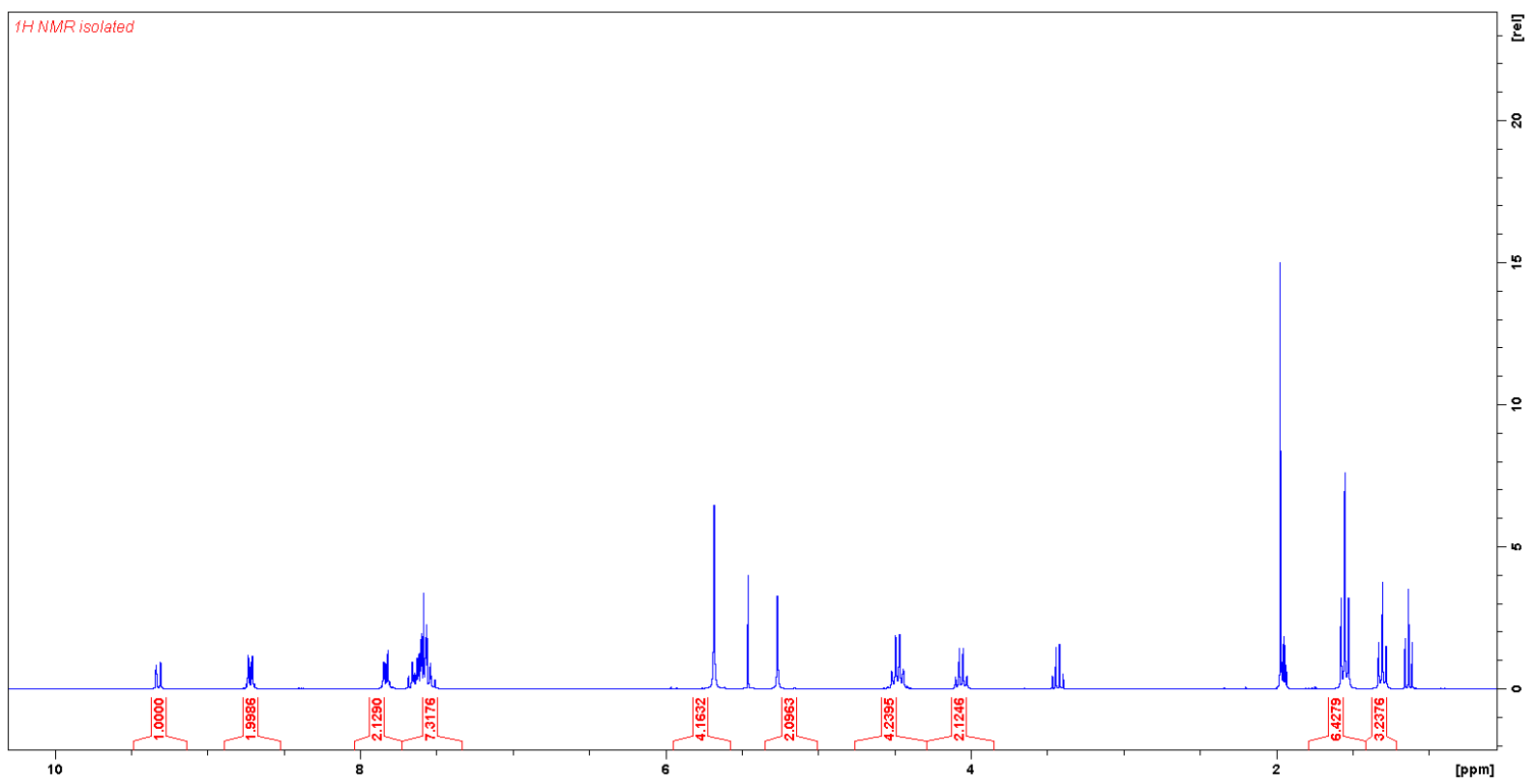

Scheme S4: ${ }^{1} \mathrm{H}$ NMR spectrum of $\left[\mathrm{GeBr}_{2}\left(\mathrm{BIMEt}_{3}\right)\right][\mathrm{OTf}]_{2}$ with signals belonging to diethyl ether (3.4 and 1.1 ppm) and for $\mathrm{CH}_{2} \mathrm{Cl}_{2}(5.44 \mathrm{ppm})$. 


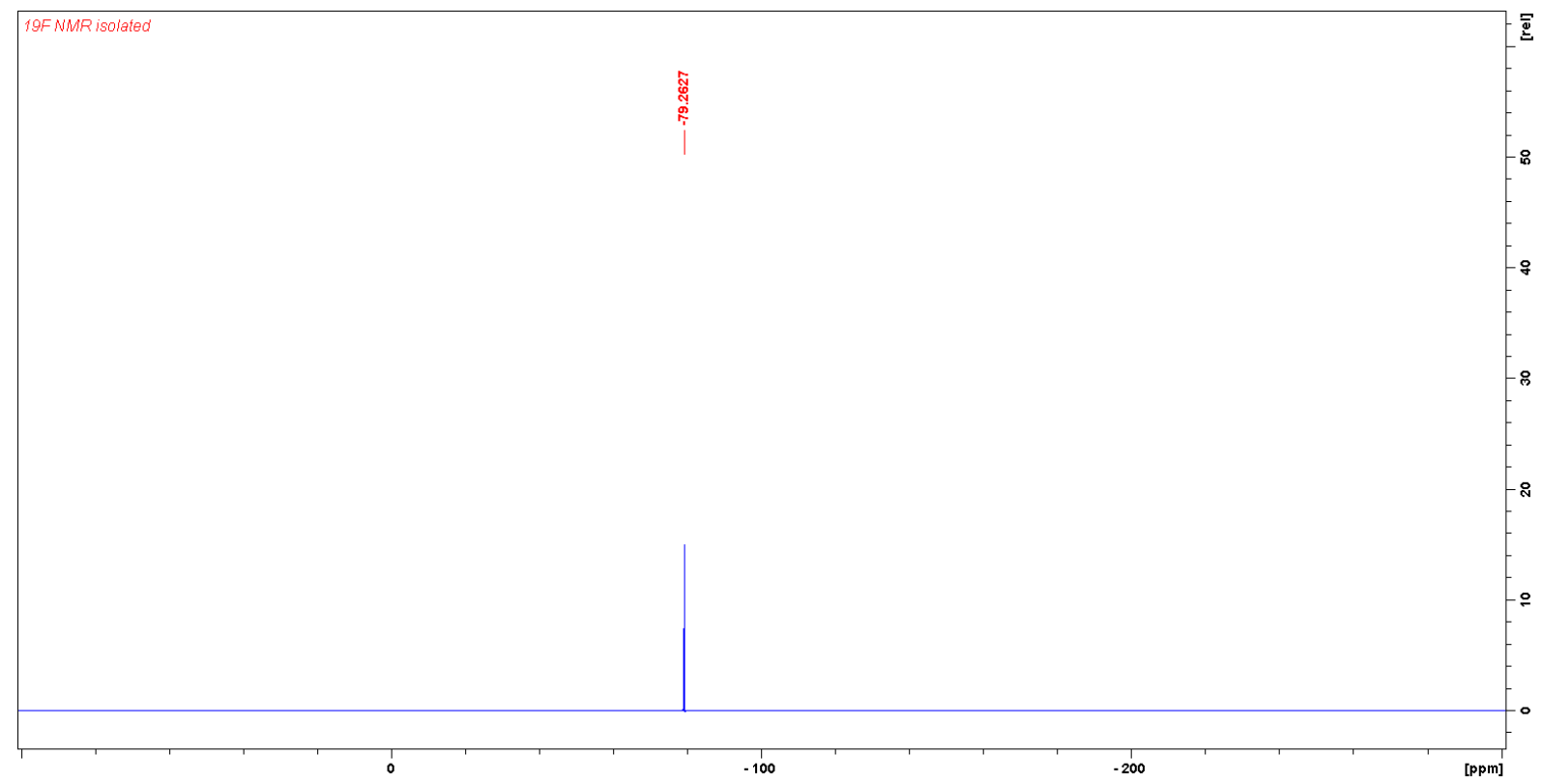

Scheme S5: ${ }^{19} \mathrm{~F}$ NMR spectrum of $\left[\mathrm{GeBr}_{2}\left(\mathrm{BIMEt}_{3}\right)\right][\mathrm{OTf}]_{2}$

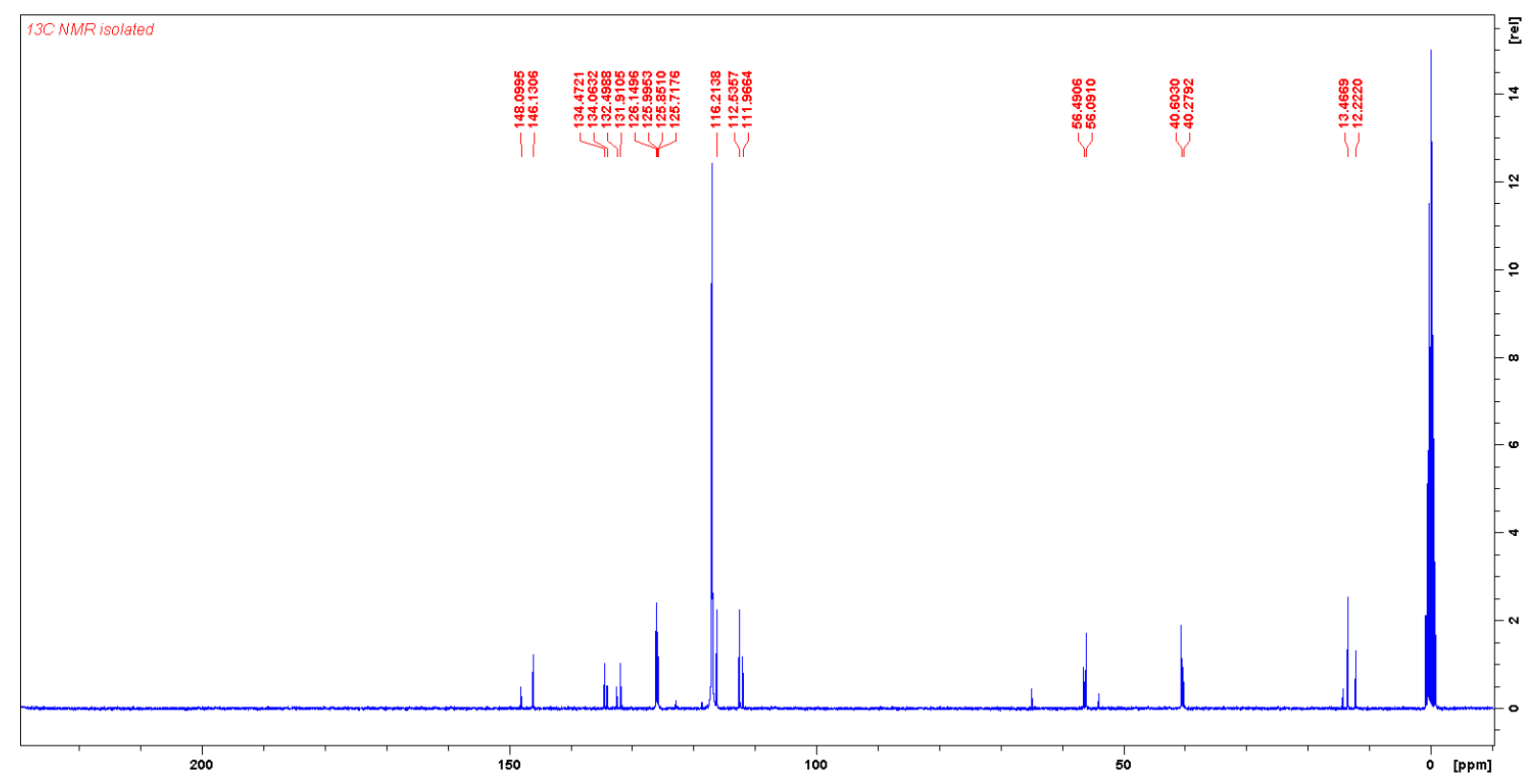

Scheme S6: ${ }^{13} \mathrm{C}$ NMR spectrum of $\left[\mathrm{GeBr}_{2}\left(\mathrm{BIMEt}_{3}\right)\right][\mathrm{OTf}]_{2}$ 
$\left[\operatorname{GeI}_{2}\left(\mathrm{BIMEt}_{3}\right)\right][\mathrm{OTf}]_{2}$

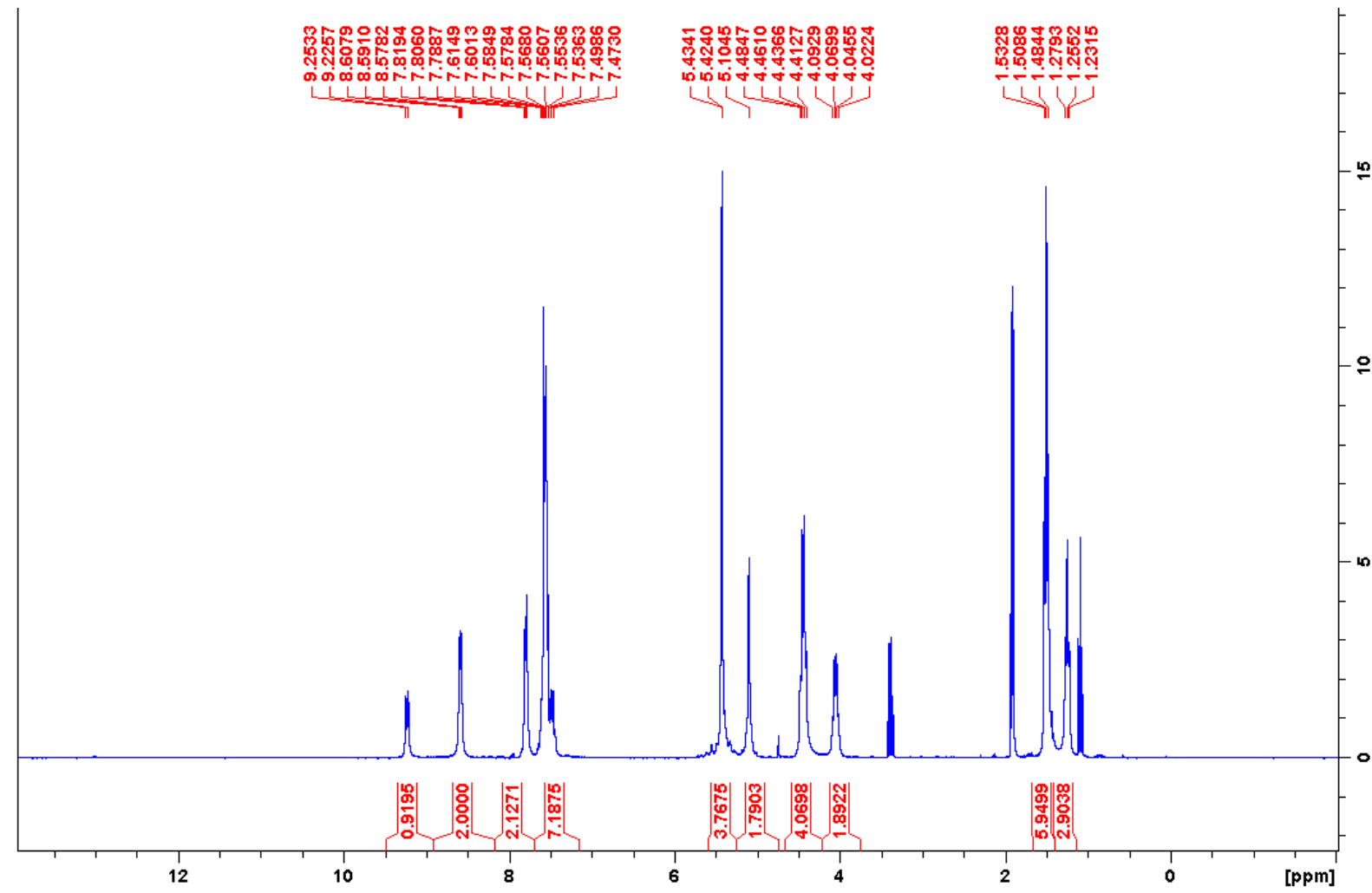

Scheme S7: ${ }^{1} \mathrm{H}$ NMR spectrum of [GeI $\left.{ }_{2}\left(\mathrm{BIMEt}_{3}\right)\right][\mathrm{OTf}]_{2}$ with signals belonging to diethyl ether (3.4 and 1.1 ppm) and for $\mathrm{CH}_{2} \mathrm{Cl}_{2}(5.44 \mathrm{ppm})$.

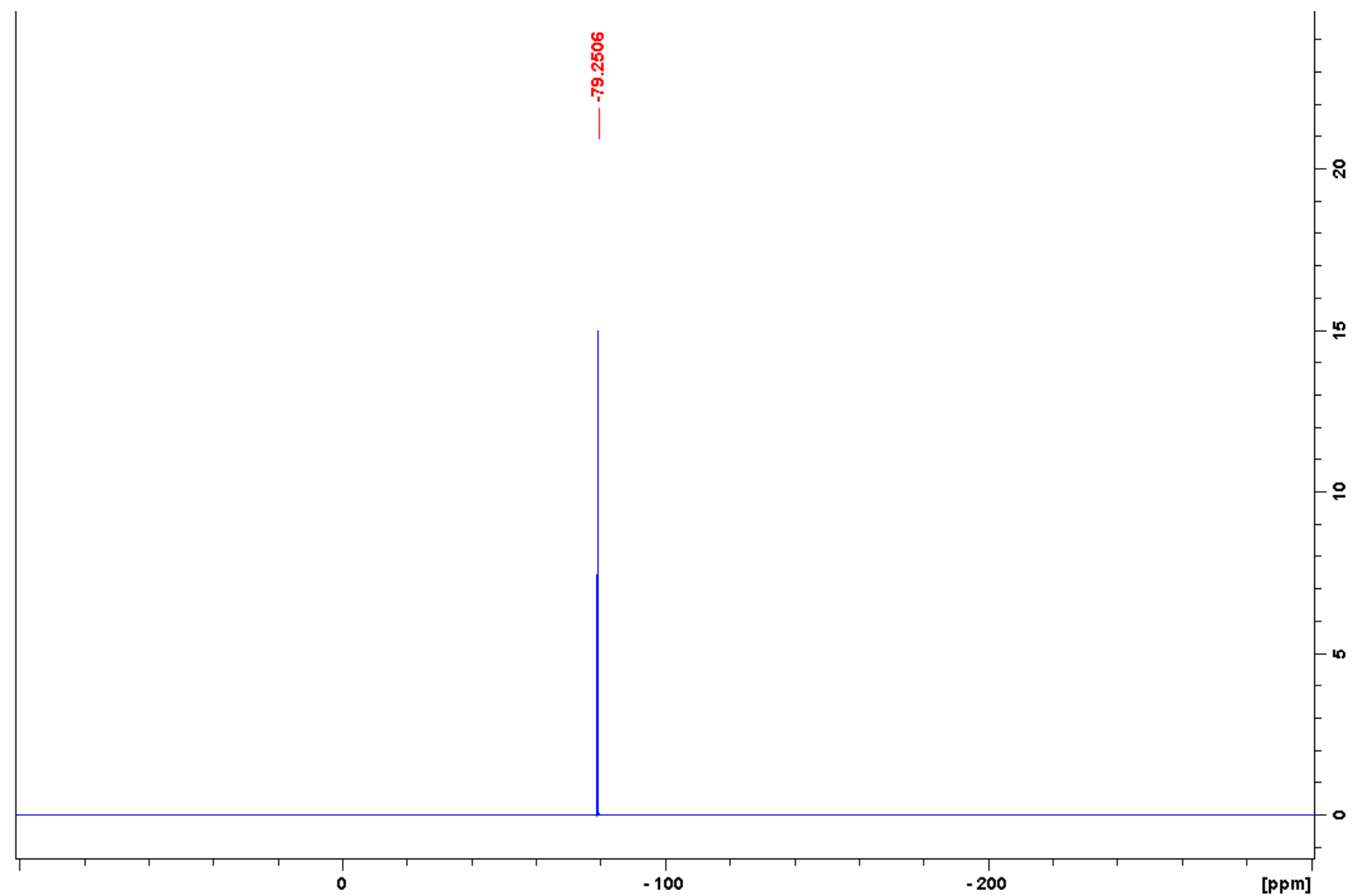

Scheme S8: ${ }^{19} \mathrm{~F}$ NMR spectrum of $\left[\mathrm{GeI}_{2}\left(\mathrm{BIMEt}_{3}\right)\right][\mathrm{OTf}]_{2}$ 


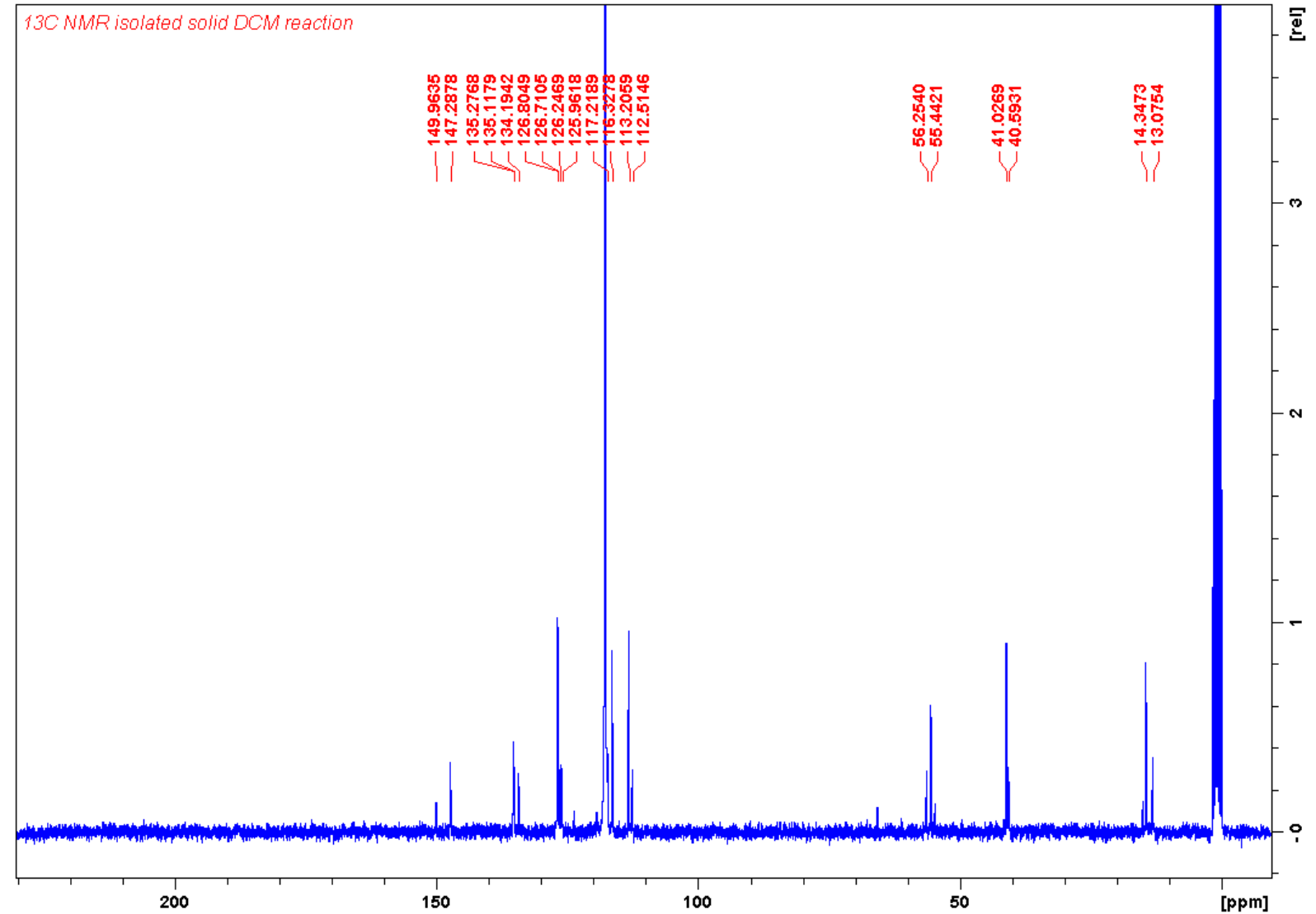

Scheme S9: ${ }^{13} \mathrm{C}$ NMR spectrum of $\left[\mathrm{GeI}_{2}\left(\mathrm{BIMEt}_{3}\right)\right][\mathrm{OTf}]_{2}$

\section{$\left[\operatorname{SnCl}_{2}\left(\right.\right.$ BIMEt $\left.\left._{3}\right)\right][\text { OTf }]_{2}$}

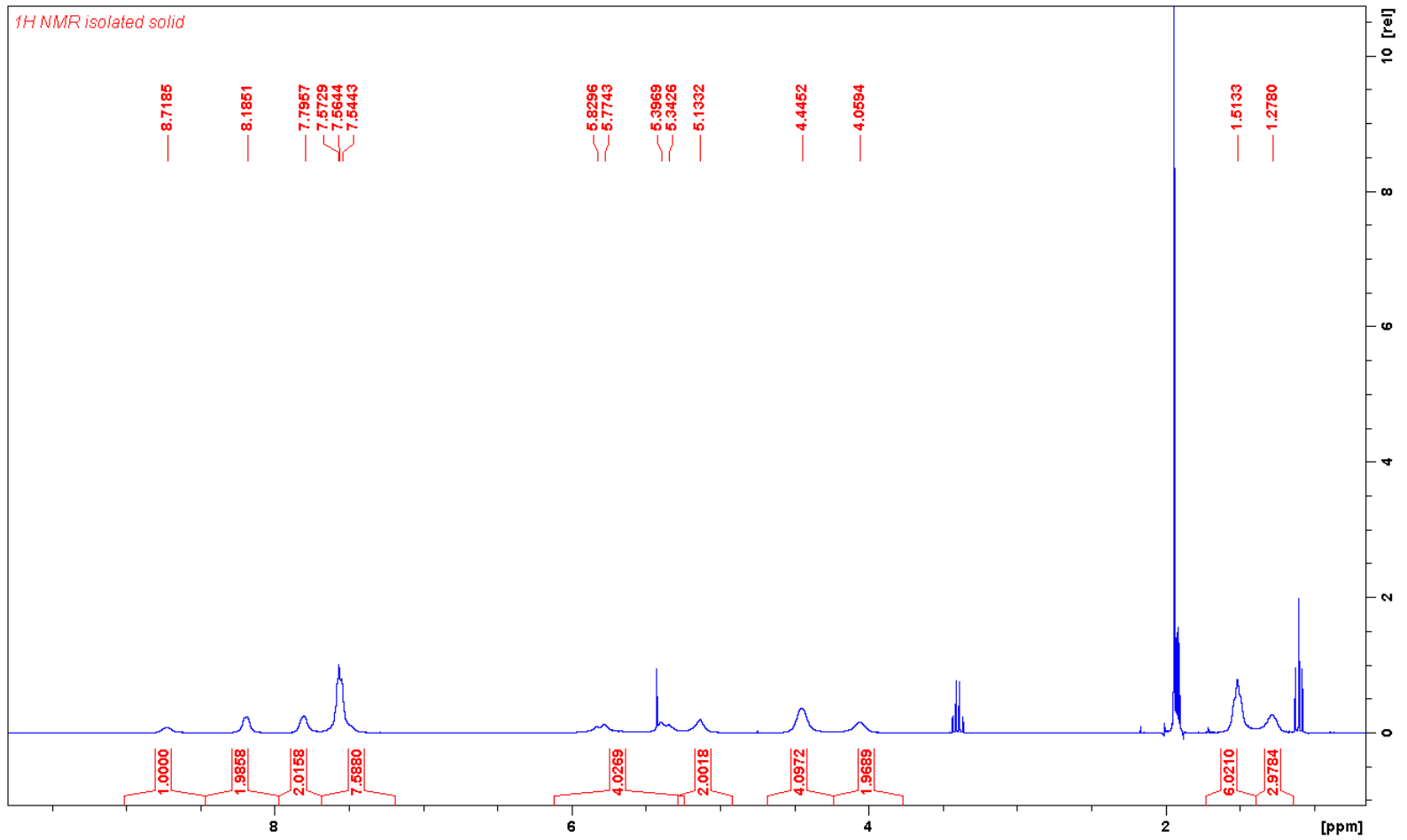

Scheme S10: ${ }^{1} \mathrm{H}$ NMR spectrum of $\left[\mathrm{SnCl}_{2}\left(\mathrm{BIMEt}_{3}\right)\right][\mathrm{OTf}]_{2}$ with signals belonging to diethyl ether (3.4 and 1.1 ppm) and for $\mathrm{CH}_{2} \mathrm{Cl}_{2}(5.44 \mathrm{ppm})$. 


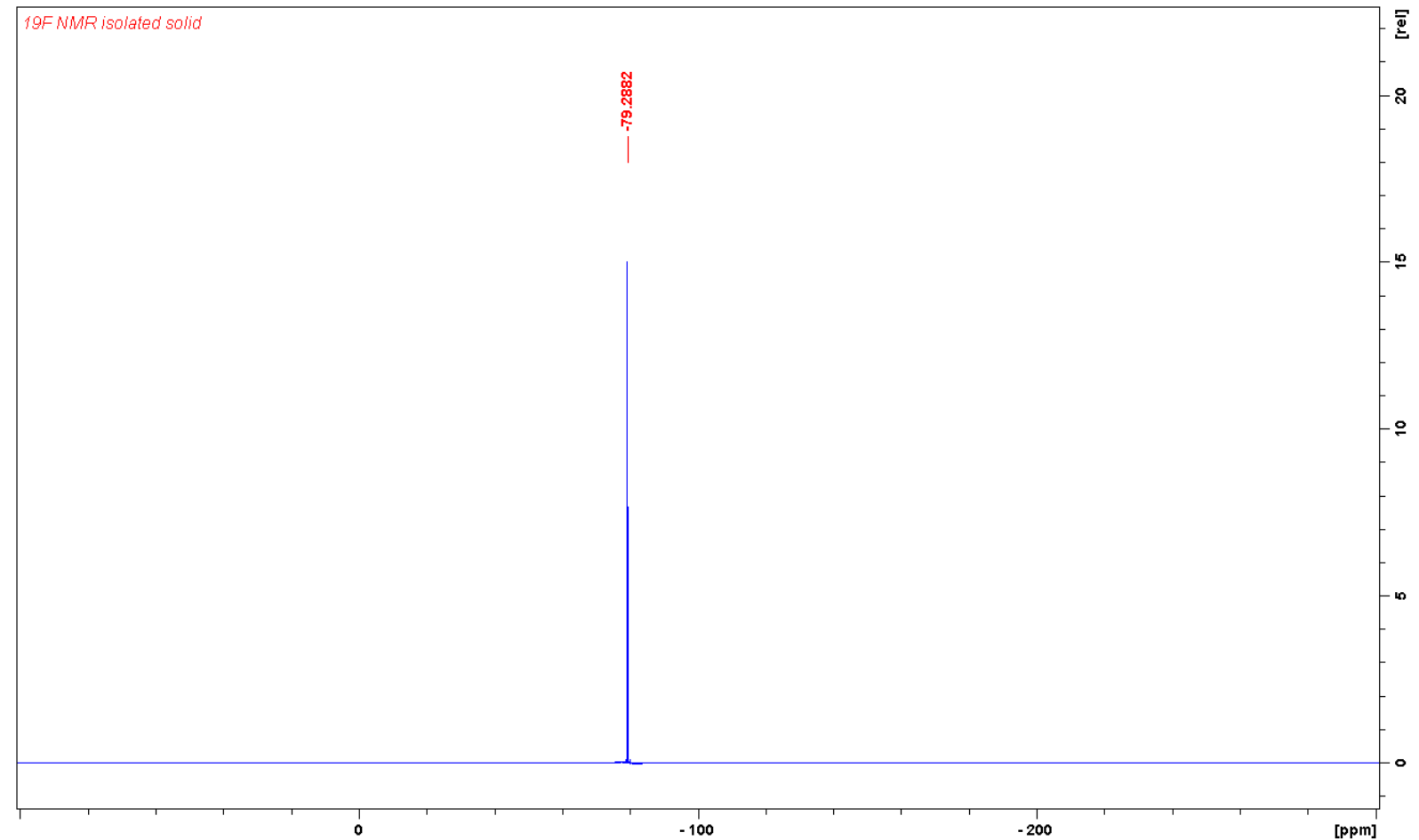

Scheme S11: ${ }^{19} \mathrm{~F}$ NMR spectrum of $\left[\mathrm{SnCl}_{2}\left(\mathrm{BIMEt}_{3}\right)\right][\mathrm{OTf}]_{2}$

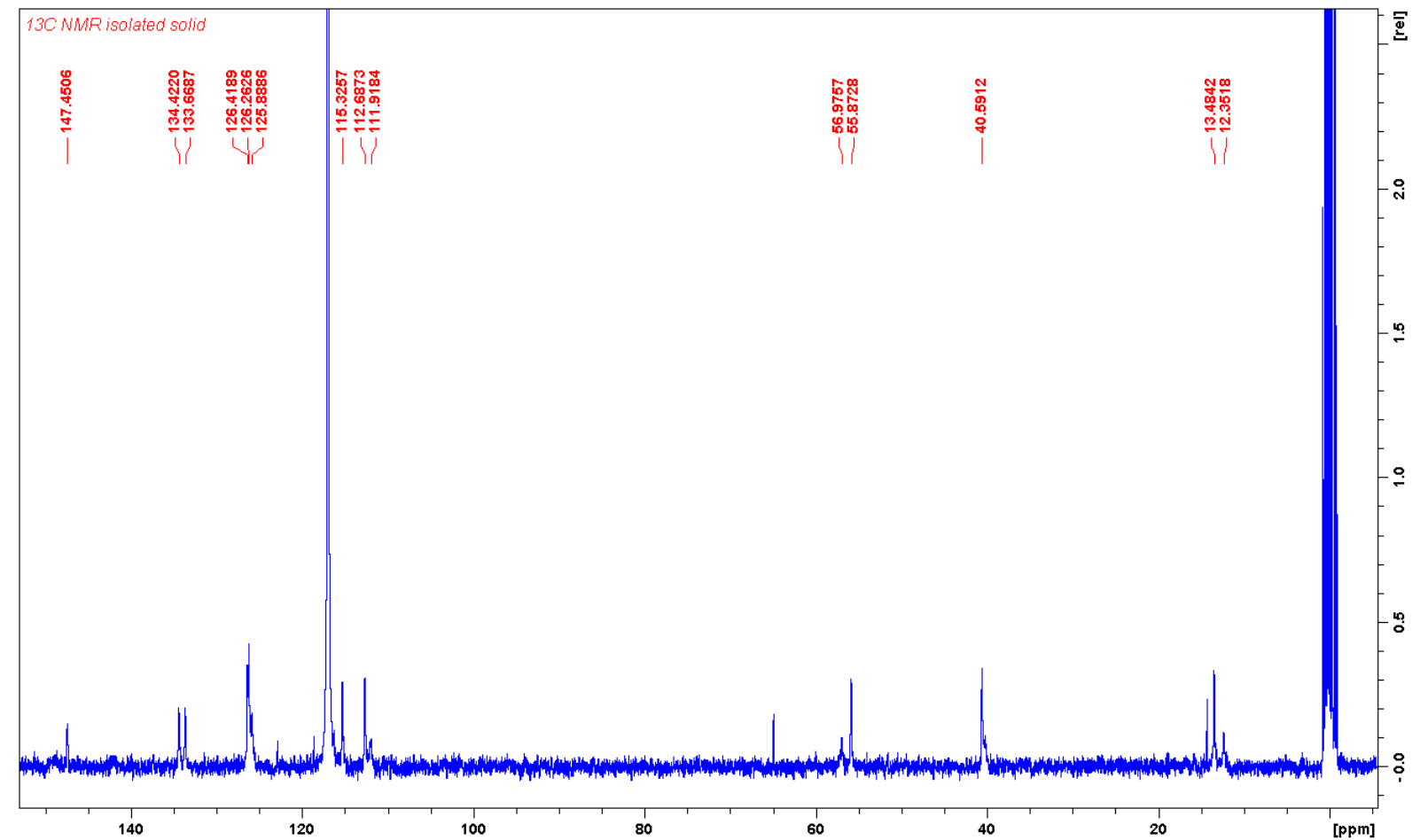

Scheme S12: ${ }^{13} \mathrm{C}$ NMR spectrum of $\left[\mathrm{SnCl}_{2}\right.$ (BIMEt 3$\left.)\right][\mathrm{OTf}]_{2}$ 


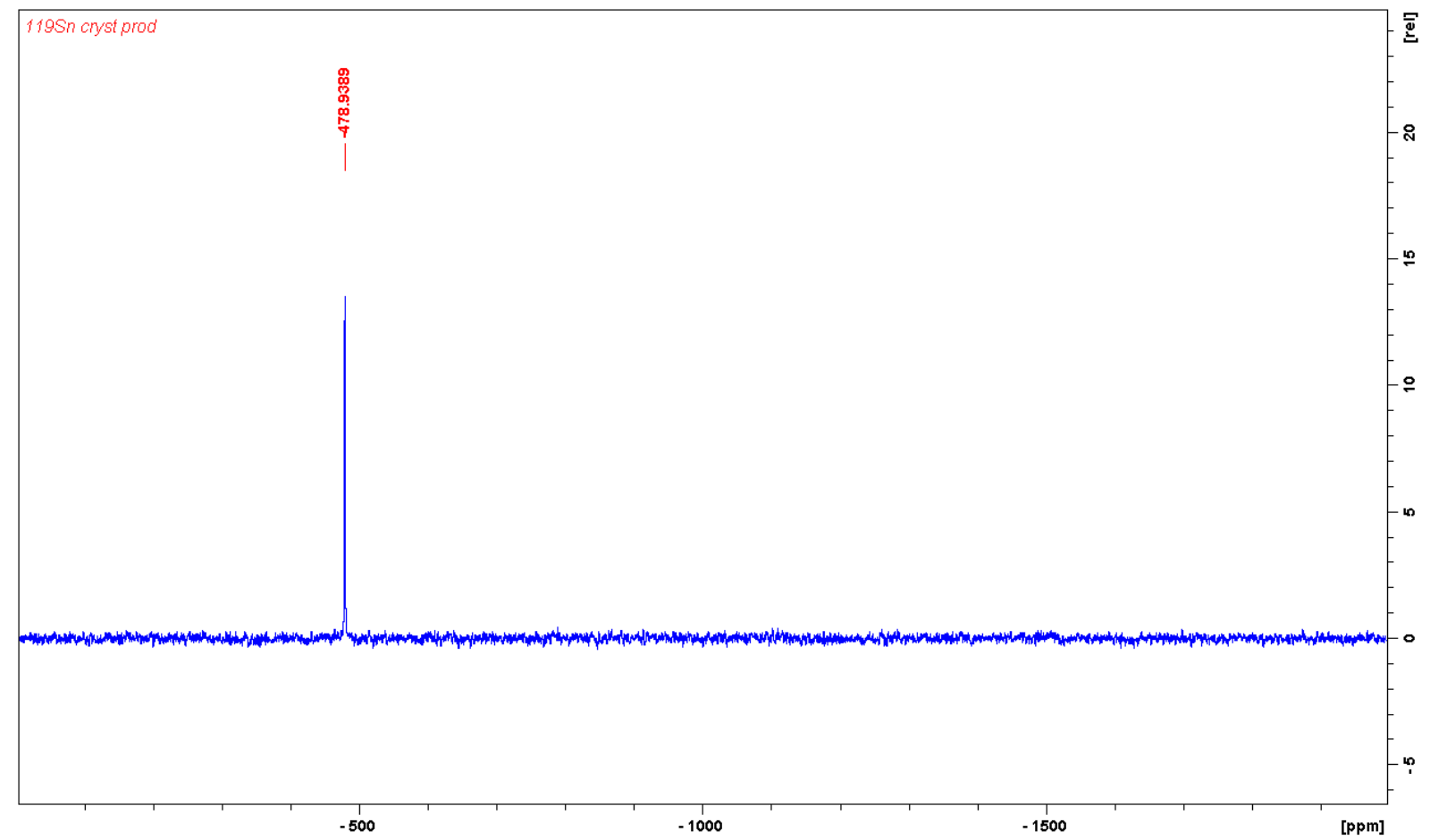

Scheme S13: ${ }^{119} \mathrm{Sn}\left\{{ }^{1} \mathrm{H}\right\}$ NMR spectrum of $\left[\mathrm{SnCl}_{2}\left(\mathrm{BIMEt}_{3}\right)\right][\mathrm{OTf}]_{2}$

[SnBr2(BIMEt3)][OTf $]_{2}$

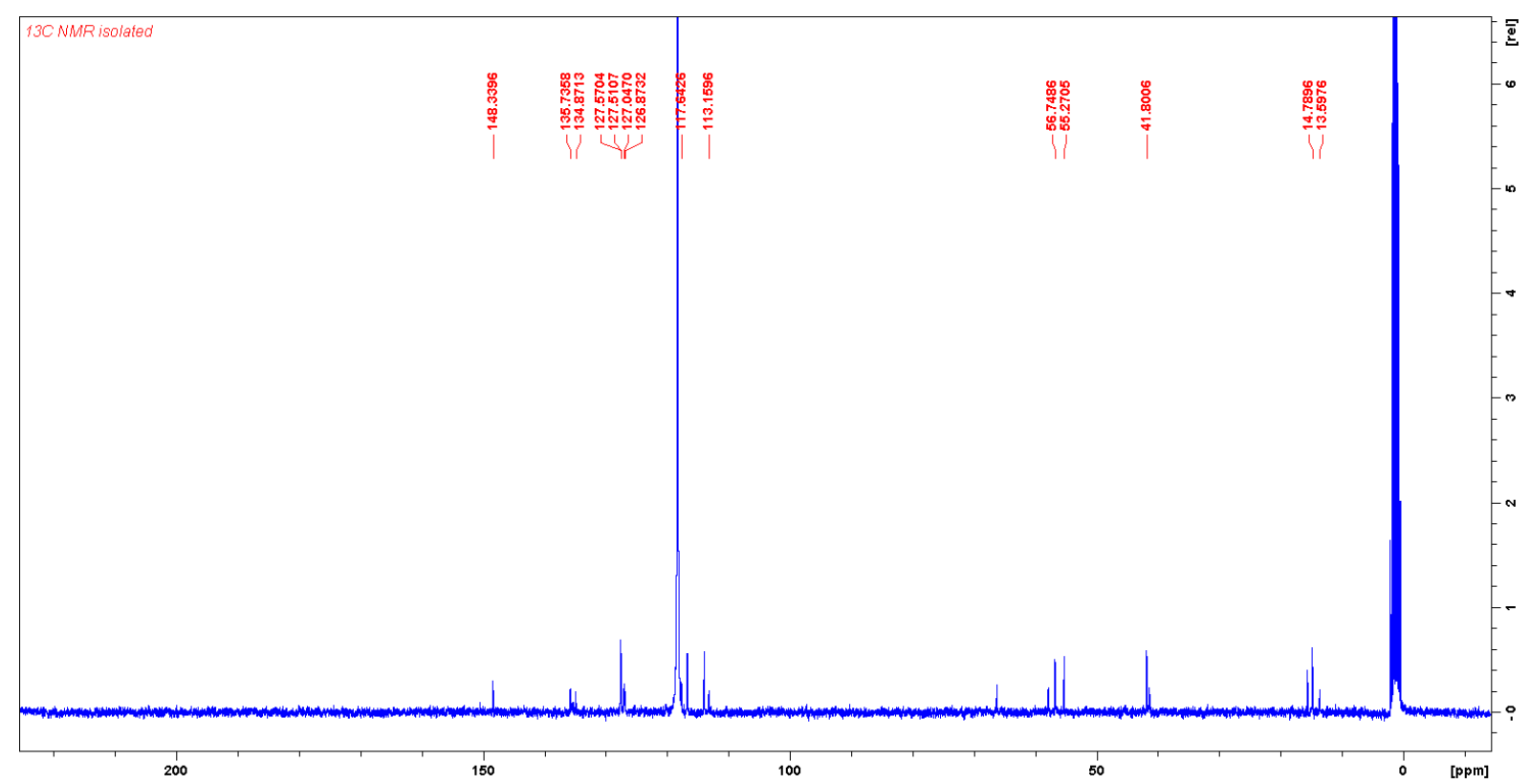

Scheme S14: ${ }^{13} \mathrm{C}$ NMR spectrum of $\left[\mathrm{SnBr}_{2}\left(\mathrm{BIMEt}_{3}\right)\right][\mathrm{OTf}]_{2}$ 


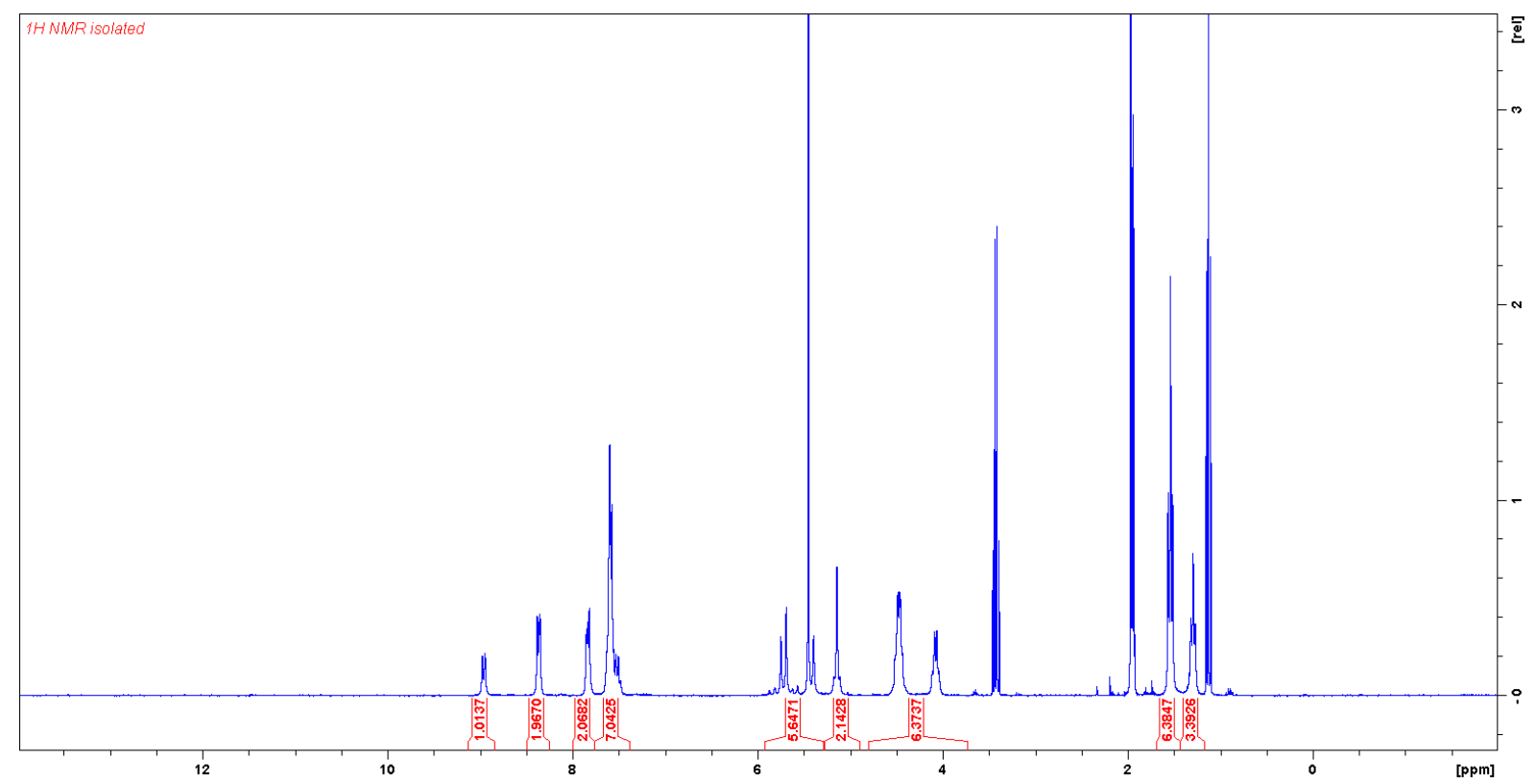

Scheme S15: ${ }^{1} \mathrm{H}$ NMR spectrum of $\left[\mathrm{SnBr}_{2}\left(\mathrm{BIMEt}_{3}\right)\right][\mathrm{OTf}]_{2}$ with signals belon ging to diethyl ether (3.4 and 1.1 ppm) and for $\mathrm{CH}_{2} \mathrm{Cl}_{2}(5.44 \mathrm{ppm})$.

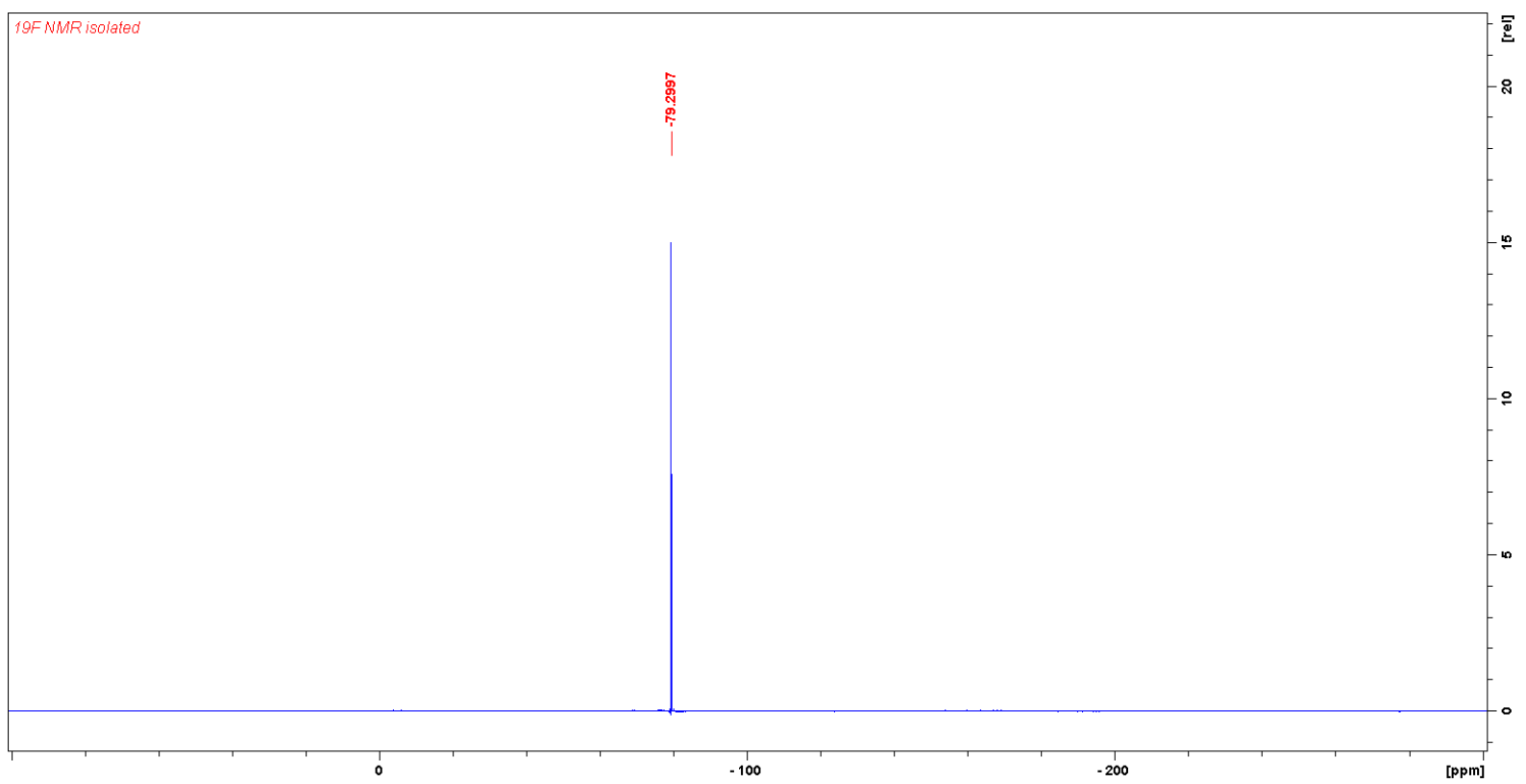

Scheme S16: ${ }^{19} \mathrm{~F}$ NMR spectrum of $\left[\mathrm{SnBr}_{2}\left(\mathrm{BIMEt}_{3}\right)\right][\mathrm{OTf}]_{2}$ 


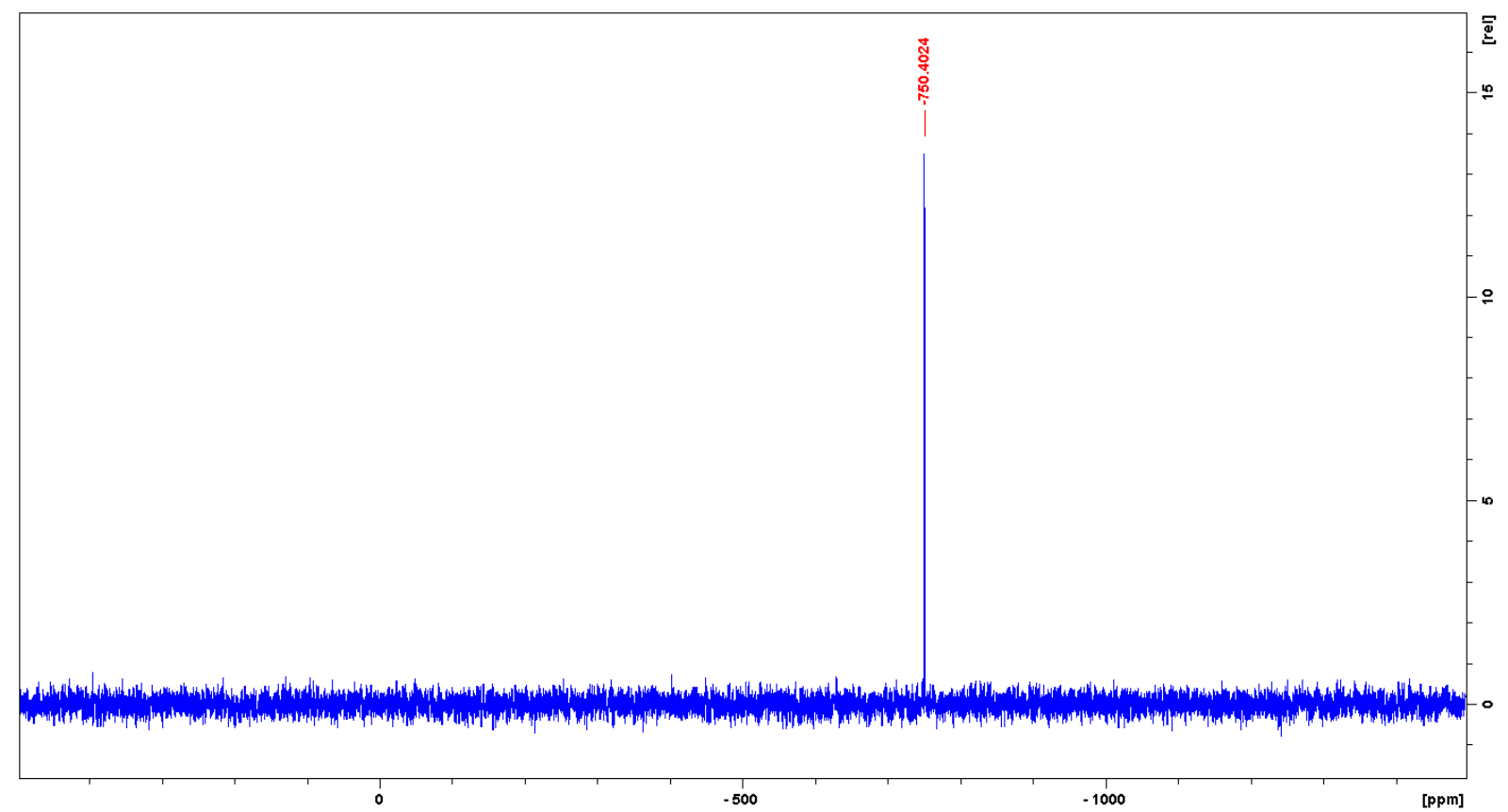

Scheme S17: ${ }^{119} \mathrm{Sn}\left\{{ }^{1} \mathrm{H}\right\}$ NMR spectrum of $\left[\mathrm{SnBr}_{2}(\mathrm{BIMEt} 3)\right][\mathrm{OTf}]_{2}$

\section{$\left[\operatorname{SnI}_{2}\left(\right.\right.$ BIMEt $\left.\left._{3}\right)\right][\text { OTf }]_{2}$}

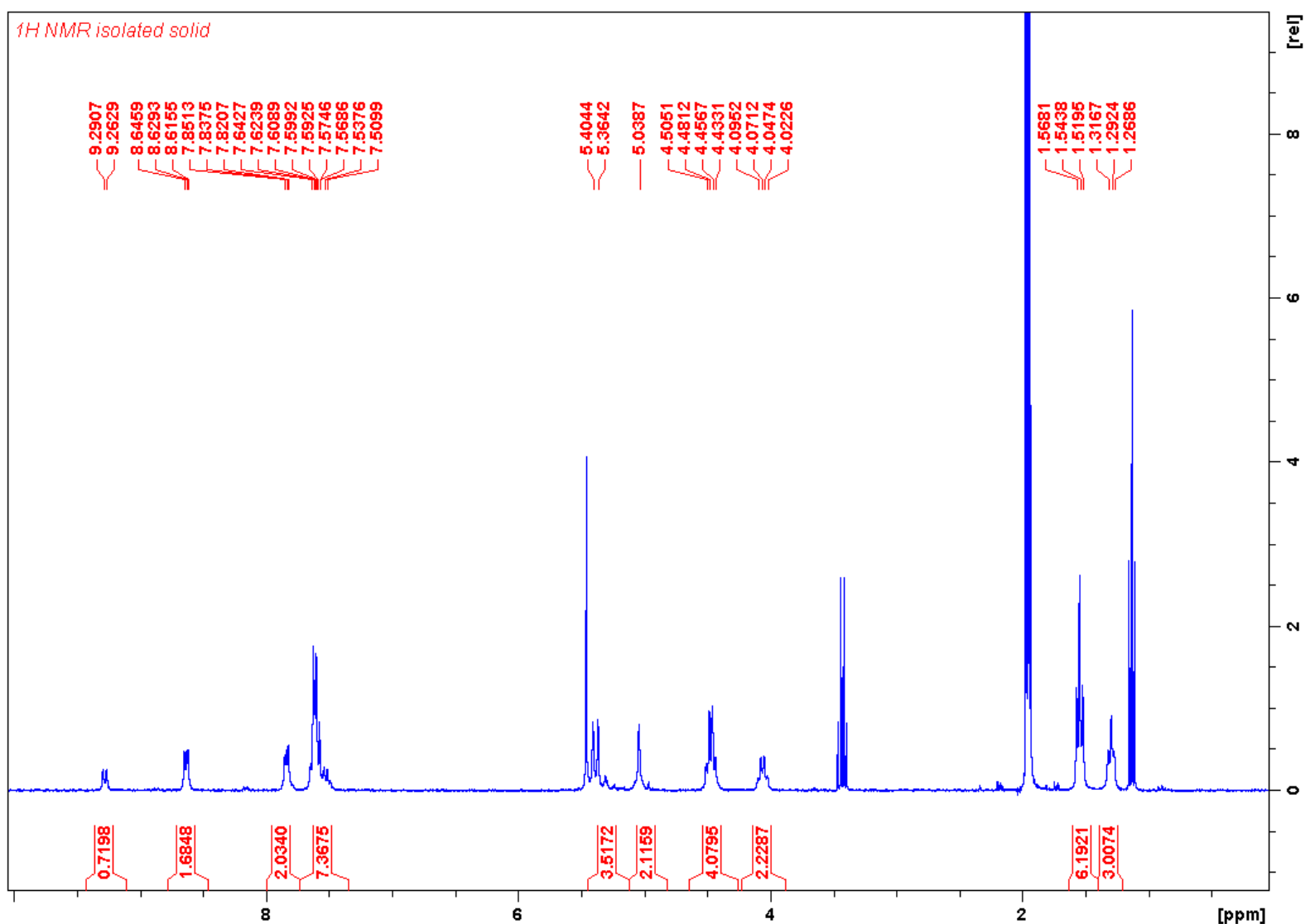

Scheme S18: ${ }^{1} \mathrm{H}$ NMR spectrum of $\left[\mathrm{SnI}_{2}\left(\mathrm{BIMEt}_{3}\right)\right][\mathrm{OTf}]_{2}$ with signals belonging to diethyl ether $(3.4$ and 1.1 ppm) and for $\mathrm{CH}_{2} \mathrm{Cl}_{2}(5.44 \mathrm{ppm})$. 


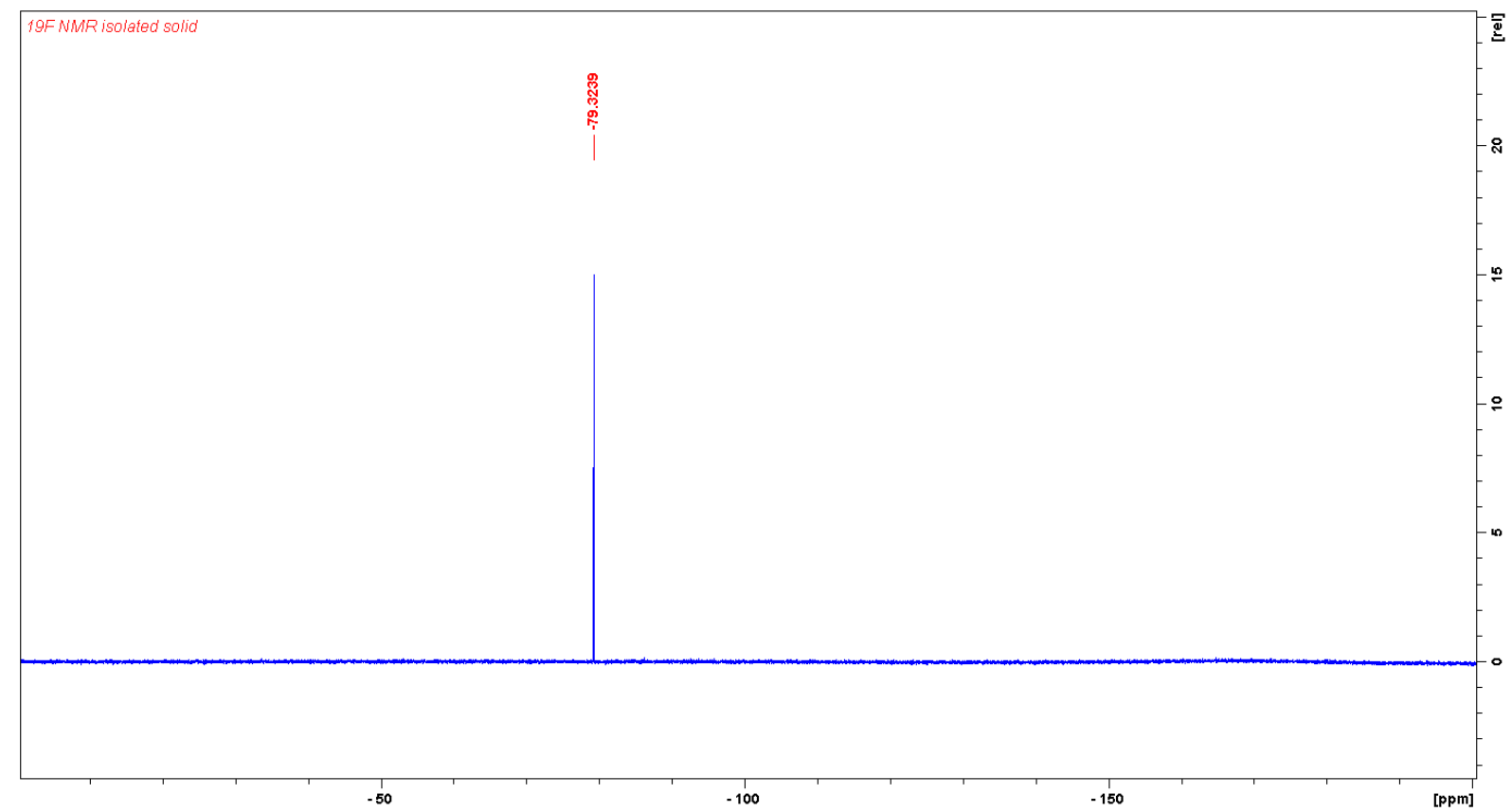

Scheme S19: ${ }^{19}$ F NMR spectrum of $\left[\mathrm{SnI}_{2}\left(\mathrm{BIMEt}_{3}\right)\right][\mathrm{OTf}]_{2}$

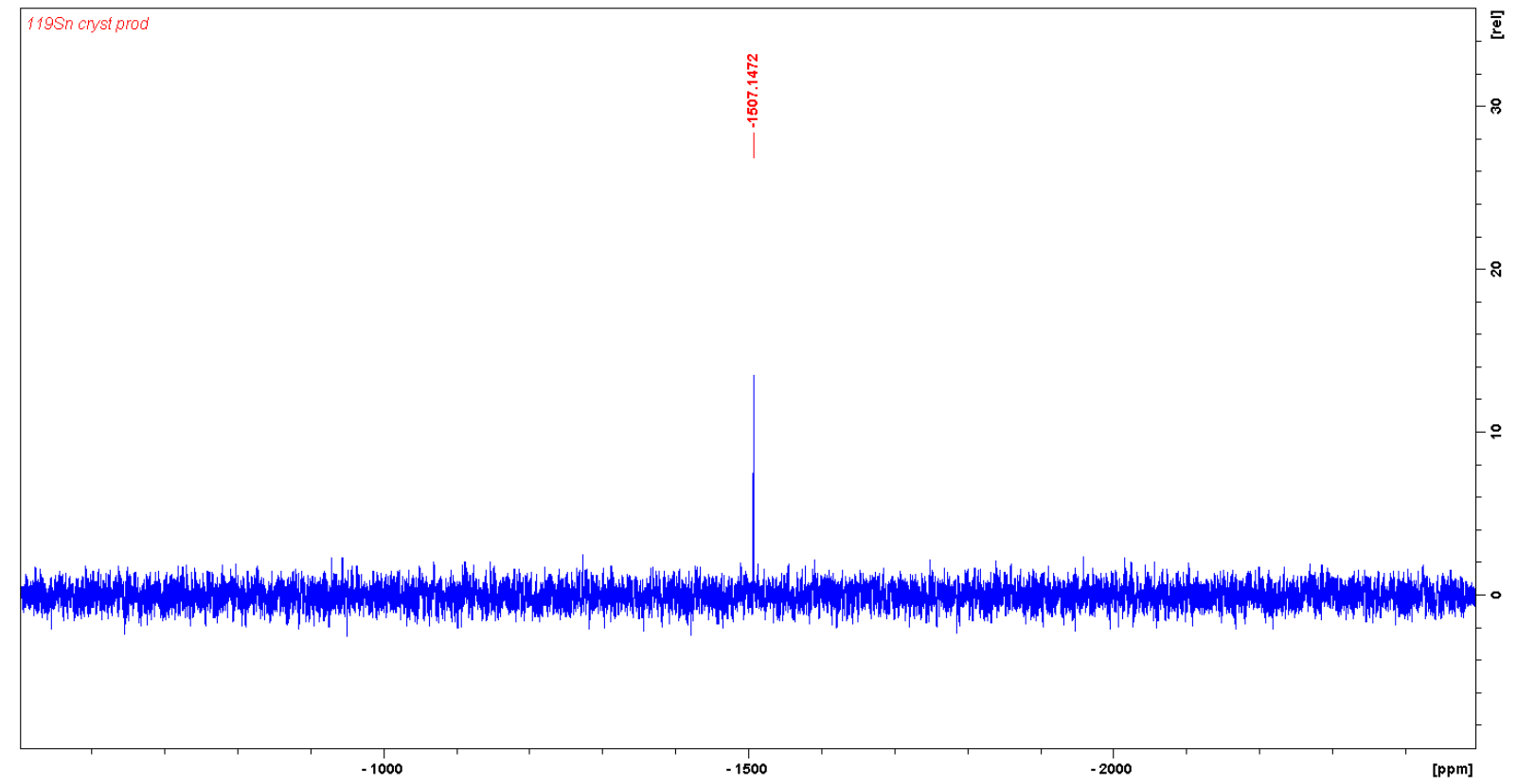

Scheme S20: ${ }^{119} \operatorname{Sn}\left\{{ }^{1} \mathrm{H}\right\}$ NMR spectrum of $\left[\mathrm{SnI}_{2}\left(\mathrm{BIMEt}_{3}\right)\right][\mathrm{OTf}]_{2}$ 
[Sn(BIMEt 3$)($ Pyr-NO) $][\text { OTf }]_{2}$

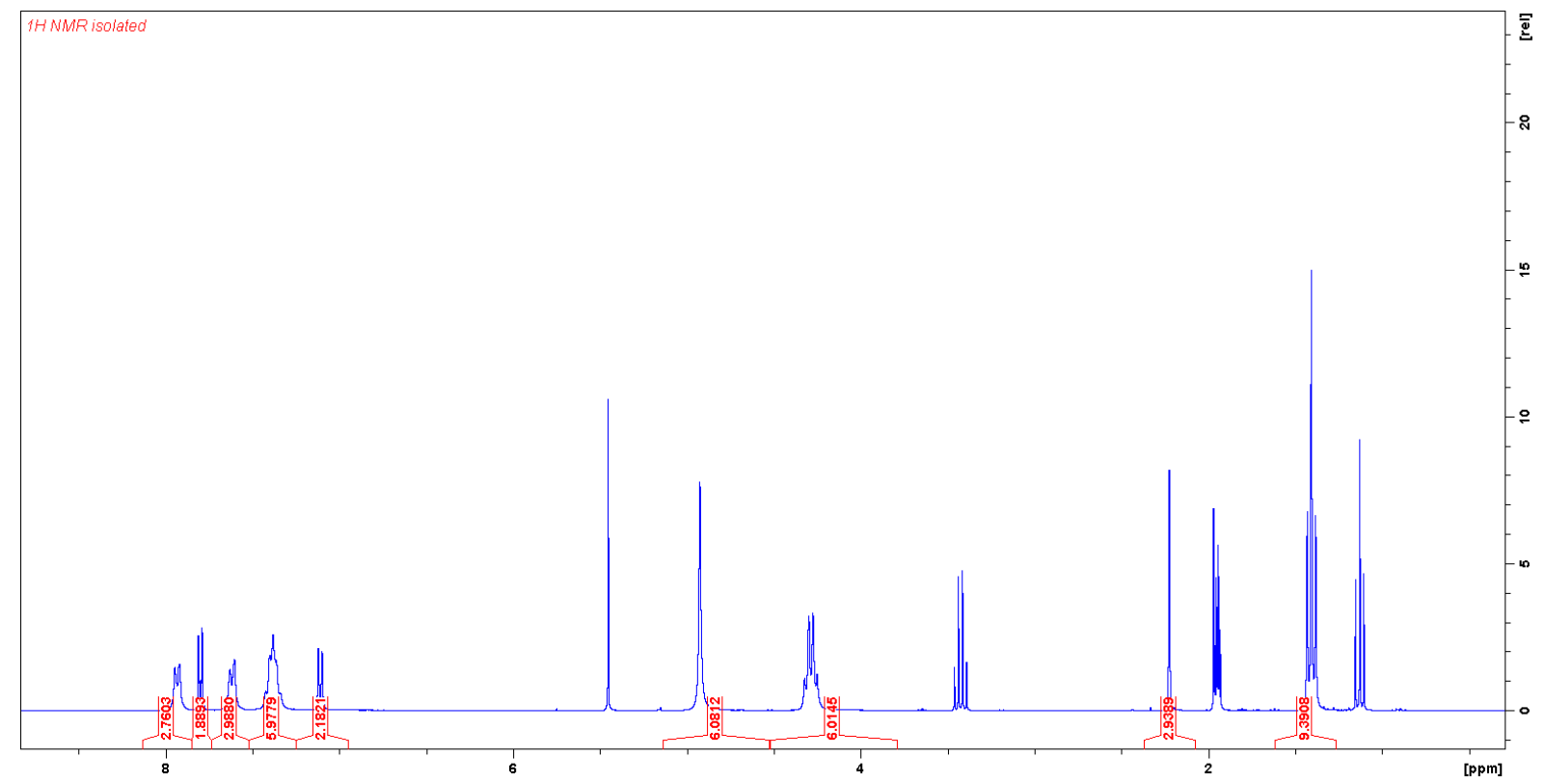

Scheme S21: ${ }^{1} \mathrm{H}$ NMR spectrum of $\left[\mathrm{Sn}\left(\mathrm{BIMEt}_{3}\right)(\mathrm{Pyr}-\mathrm{NO})\right][\mathrm{OTf}]_{2}$ with signals belonging to diethyl ether $(3.4$ and $1.1 \mathrm{ppm})$ and for $\mathrm{CH}_{2} \mathrm{Cl}_{2}(5.44 \mathrm{ppm})$.

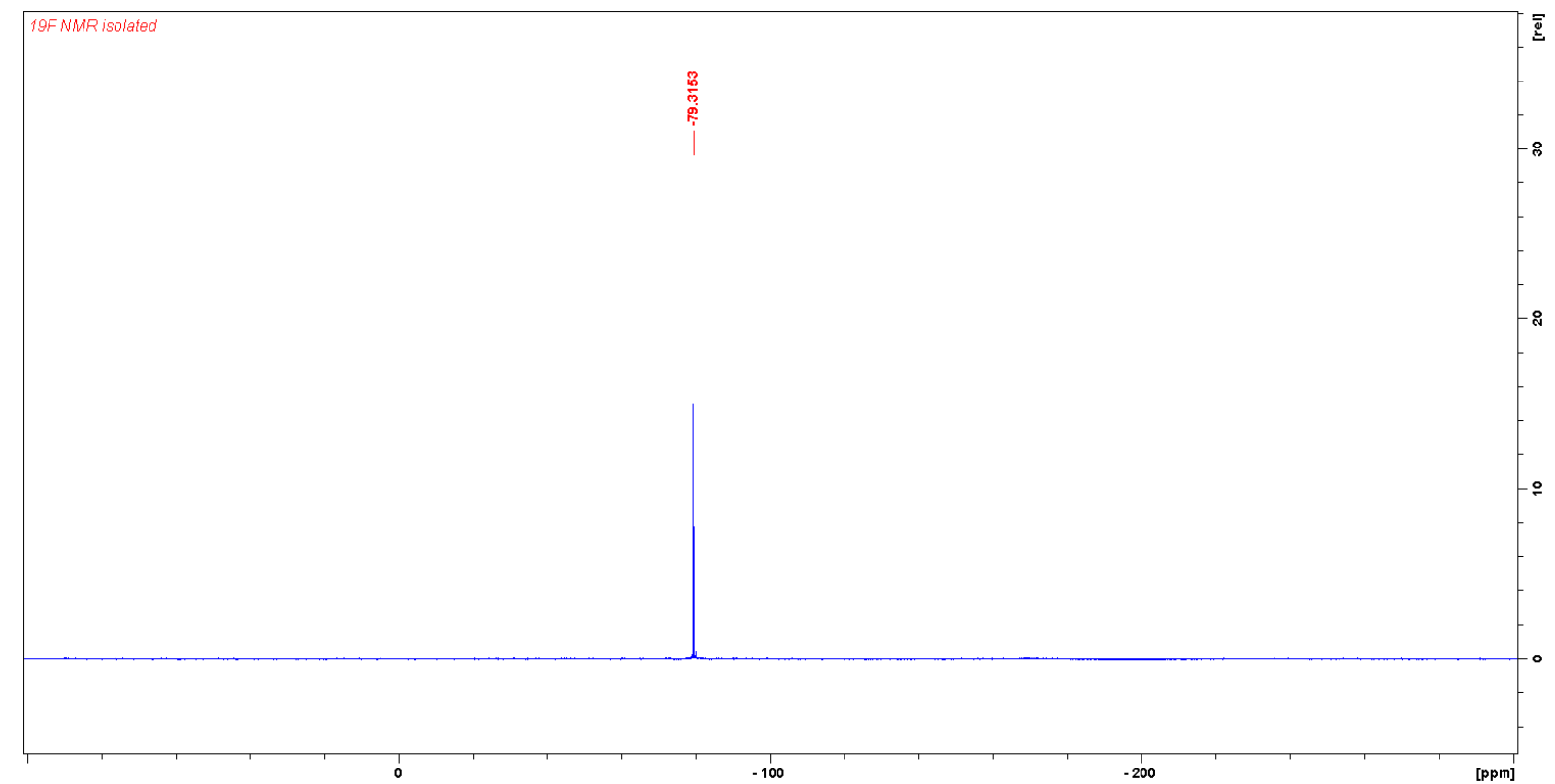

Scheme S22: ${ }^{19} \mathrm{~F}$ NMR spectrum of $\left[\mathrm{Sn}\left(\mathrm{BIMEt}_{3}\right)(\mathrm{Pyr}-\mathrm{NO})\right][\mathrm{OTf}]_{2}$ 


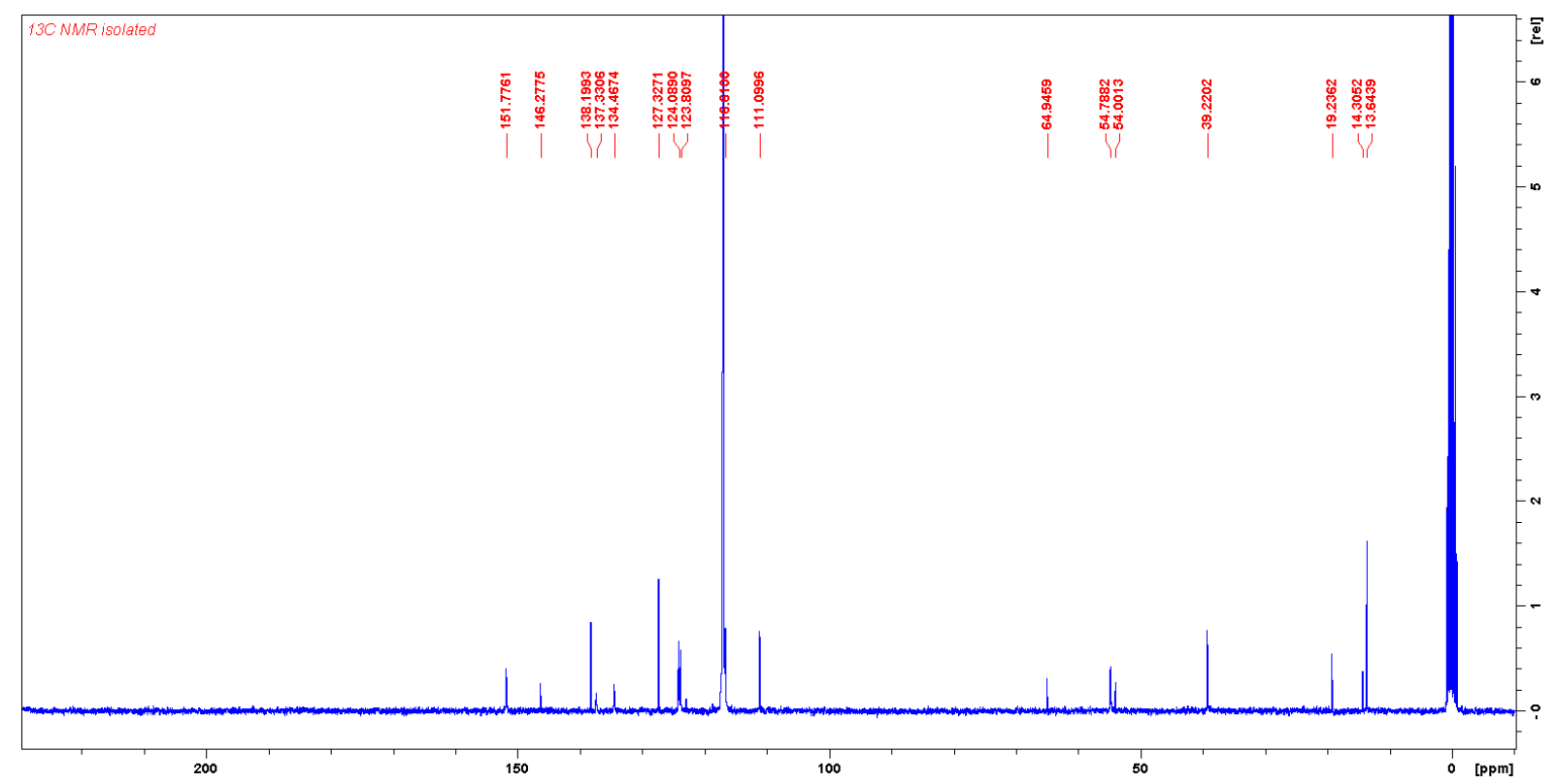

Scheme S23: ${ }^{13} \mathrm{C}$ NMR spectrum of $\left[\mathrm{Sn}\left(\mathrm{BIMEt}_{3}\right)(\mathrm{Pyr}-\mathrm{NO})\right][\mathrm{OTf}]_{2}$

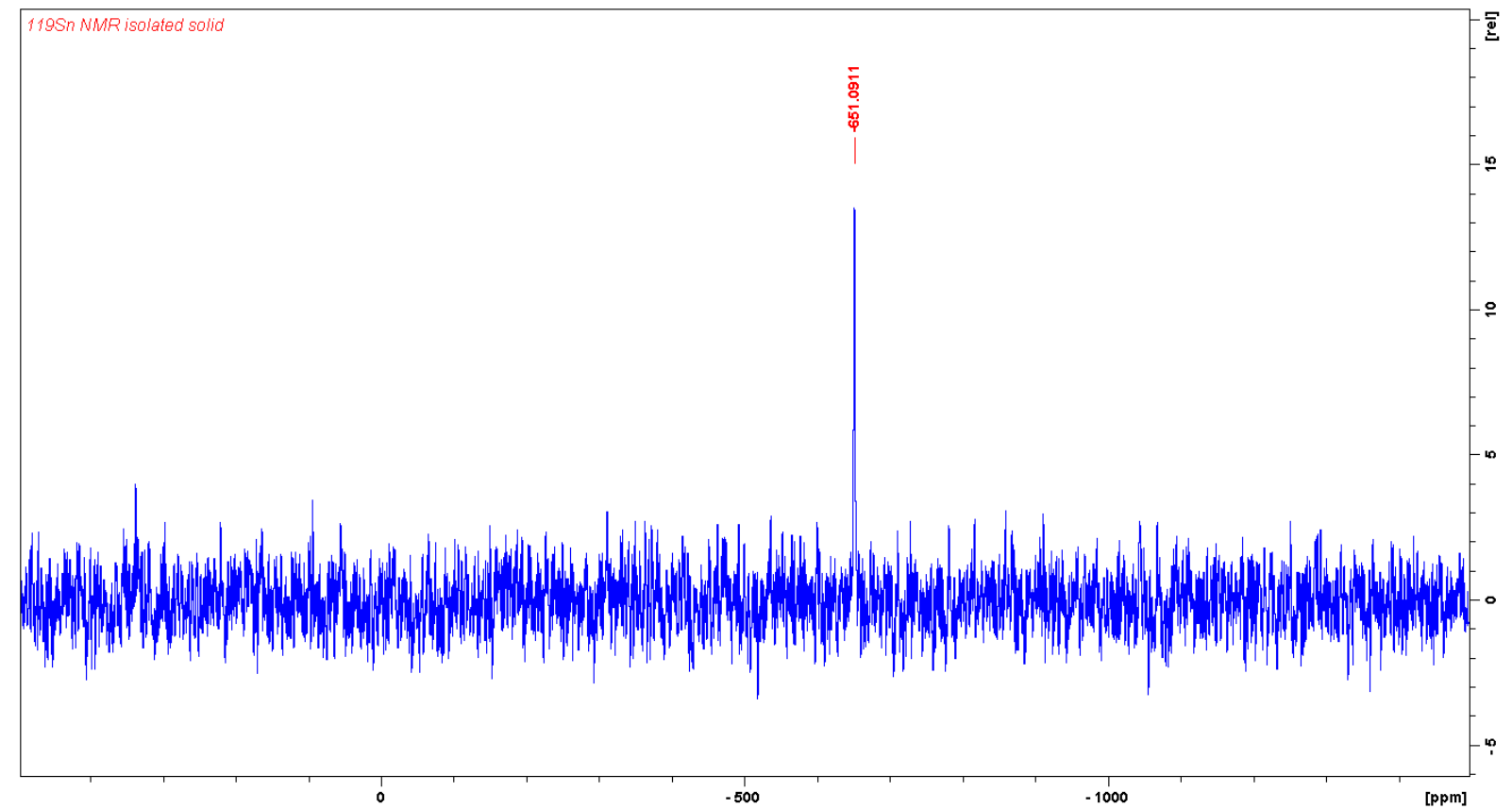

Scheme S24: ${ }^{119} \mathrm{Sn}\left\{{ }^{1} \mathrm{H}\right\}$ NMR spectrum of $\left[\mathrm{Sn}\left(\mathrm{BIMEt}_{3}\right)(\mathrm{Pyr}-\mathrm{NO})\right][\mathrm{OTf}]_{2}$ 
[Ge(BIMEt 3$)($ Pyr-NO) $][\text { OTf }]_{2}$

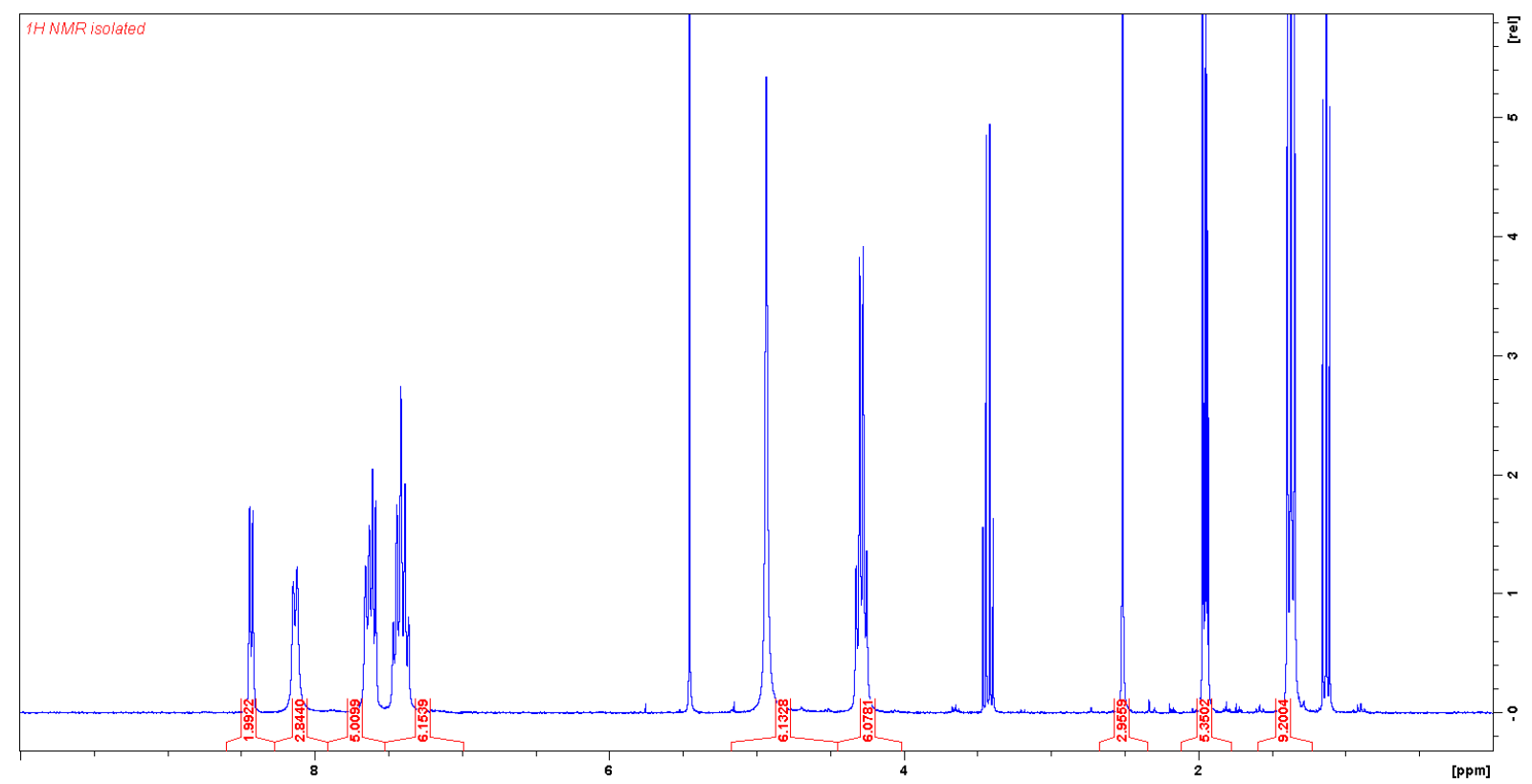

Scheme S25: ${ }^{1} \mathrm{H}$ NMR spectrum of $\left[\mathrm{Ge}\left(\mathrm{BIMEt}_{3}\right)(\mathrm{Pyr}-\mathrm{NO})\right][\mathrm{OTf}]_{2}$ with signals belonging to diethyl ether (3.4 and $1.1 \mathrm{ppm})$ and for $\mathrm{CH}_{2} \mathrm{Cl}_{2}(5.44 \mathrm{ppm})$.

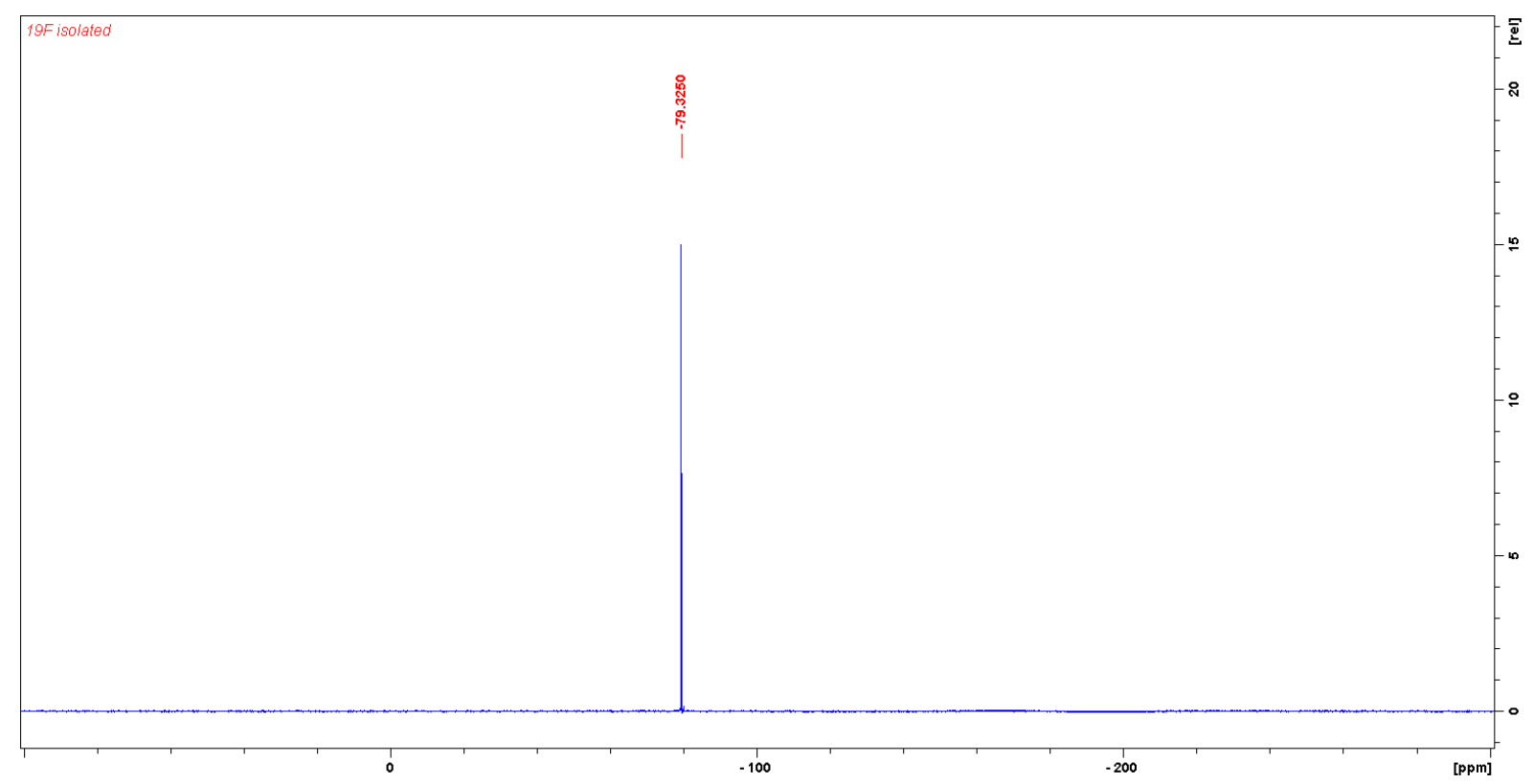

Scheme S26: ${ }^{19} \mathrm{~F}$ NMR spectrum of $\left[\mathrm{Ge}\left(\mathrm{BIMEt}_{3}\right)(\mathrm{Pyr}-\mathrm{NO})\right][\mathrm{OTf}]_{2}$ 


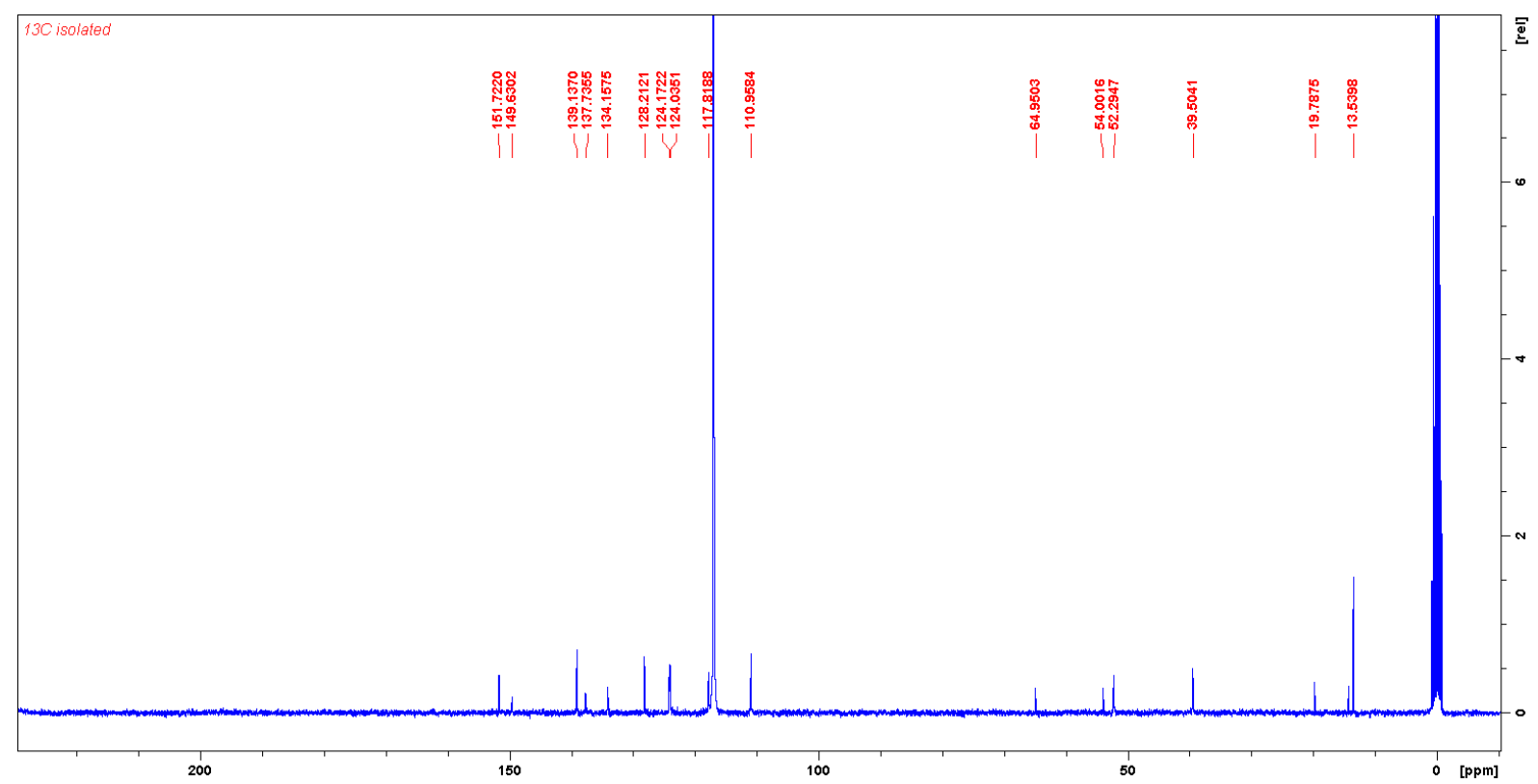

Scheme S27: ${ }^{13} \mathrm{C}$ NMR spectrum of $\left[\mathrm{Ge}\left(\mathrm{BIMEt}_{3}\right)(\mathrm{Pyr}-\mathrm{NO})\right][\mathrm{OTf}]_{2}$

\section{$\left[\operatorname{Sn}\left(\right.\right.$ OPEt $\left._{3}\right)\left(\right.$ BIMEt $\left.\left._{3}\right)\right][\text { OTf }]_{2}$}

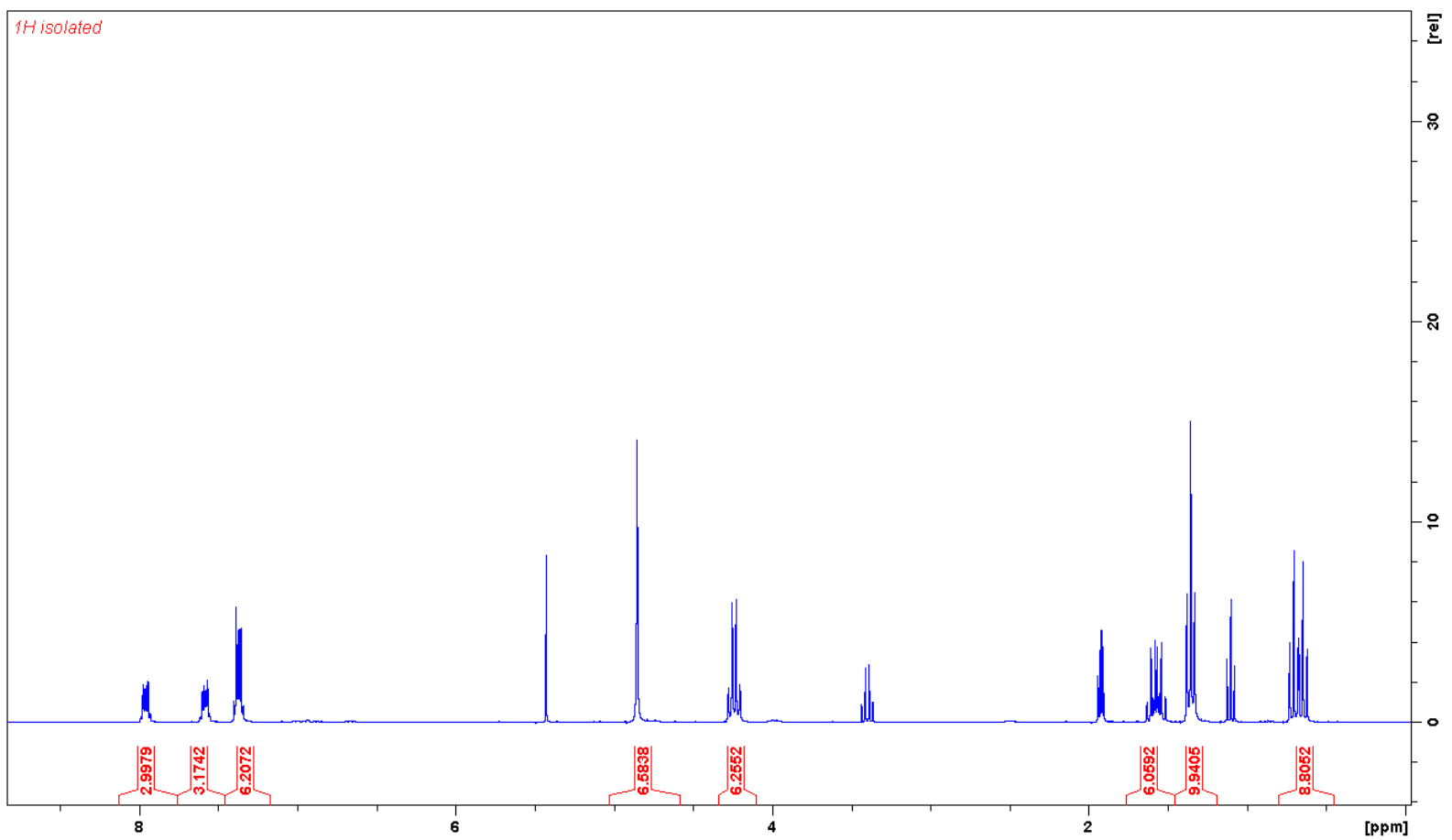

Scheme S28: ${ }^{1} \mathrm{H}$ NMR spectrum of $\left[\mathrm{Sn}\left(\mathrm{OPEt}_{3}\right)\left(\mathrm{BIMEt}_{3}\right)\right][\mathrm{OTf}]_{2}$ with signals belonging to diethyl ether (3.4 and $1.1 \mathrm{ppm})$ and for $\mathrm{CH}_{2} \mathrm{Cl}_{2}(5.44 \mathrm{ppm})$. 


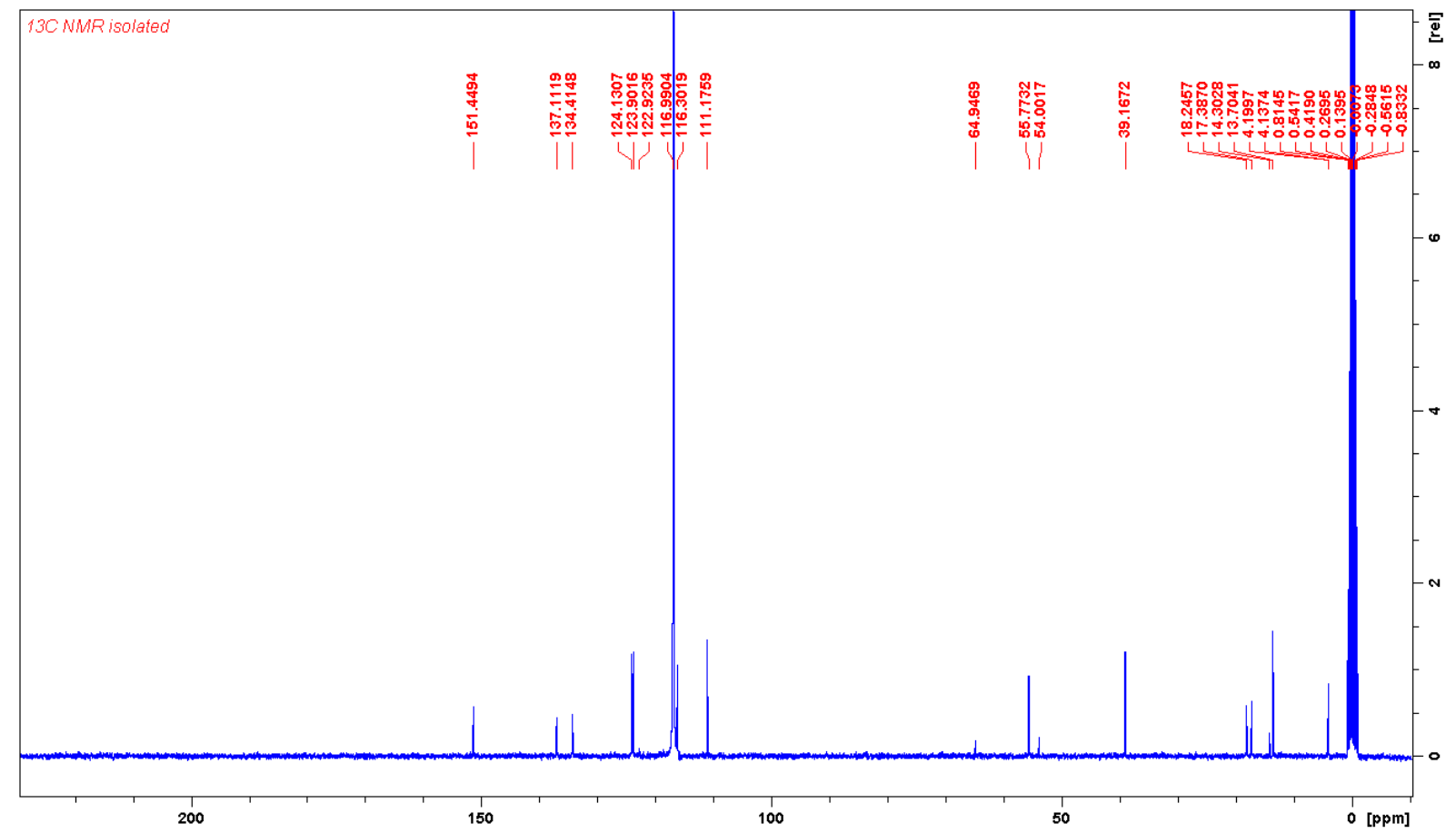

Scheme S29: ${ }^{13} \mathrm{C}$ NMR spectrum of [ $\left.\mathrm{Sn}\left(\mathrm{OPEt}_{3}\right)\left(\mathrm{BIMEt}_{3}\right)\right][\mathrm{OTf}]_{2}$

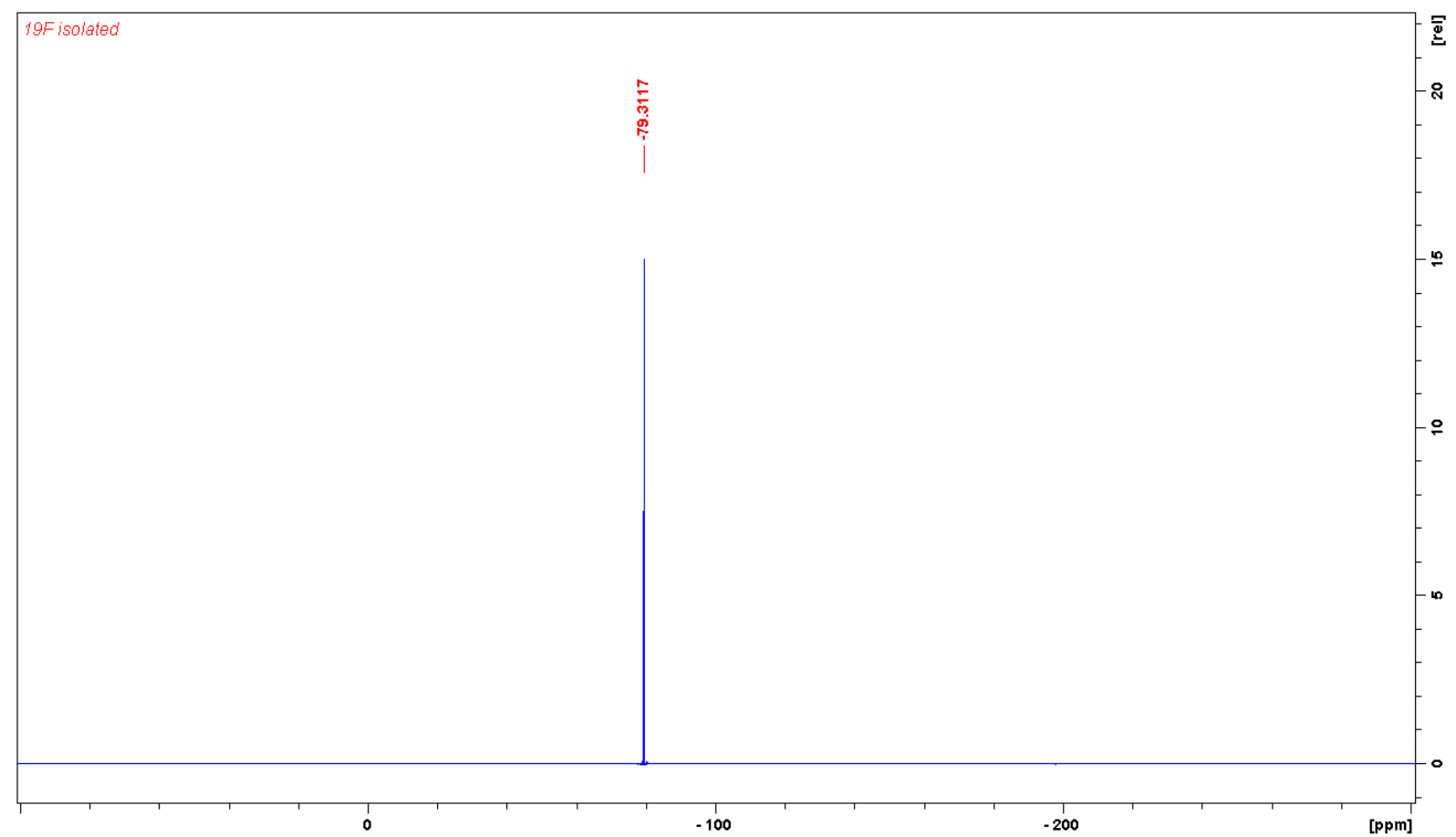

Scheme S30: ${ }^{19} \mathrm{~F}$ NMR spectrum of [Sn(OPEt3)(BIMEt 3$\left.)\right][\mathrm{OTf}]_{2}$ 


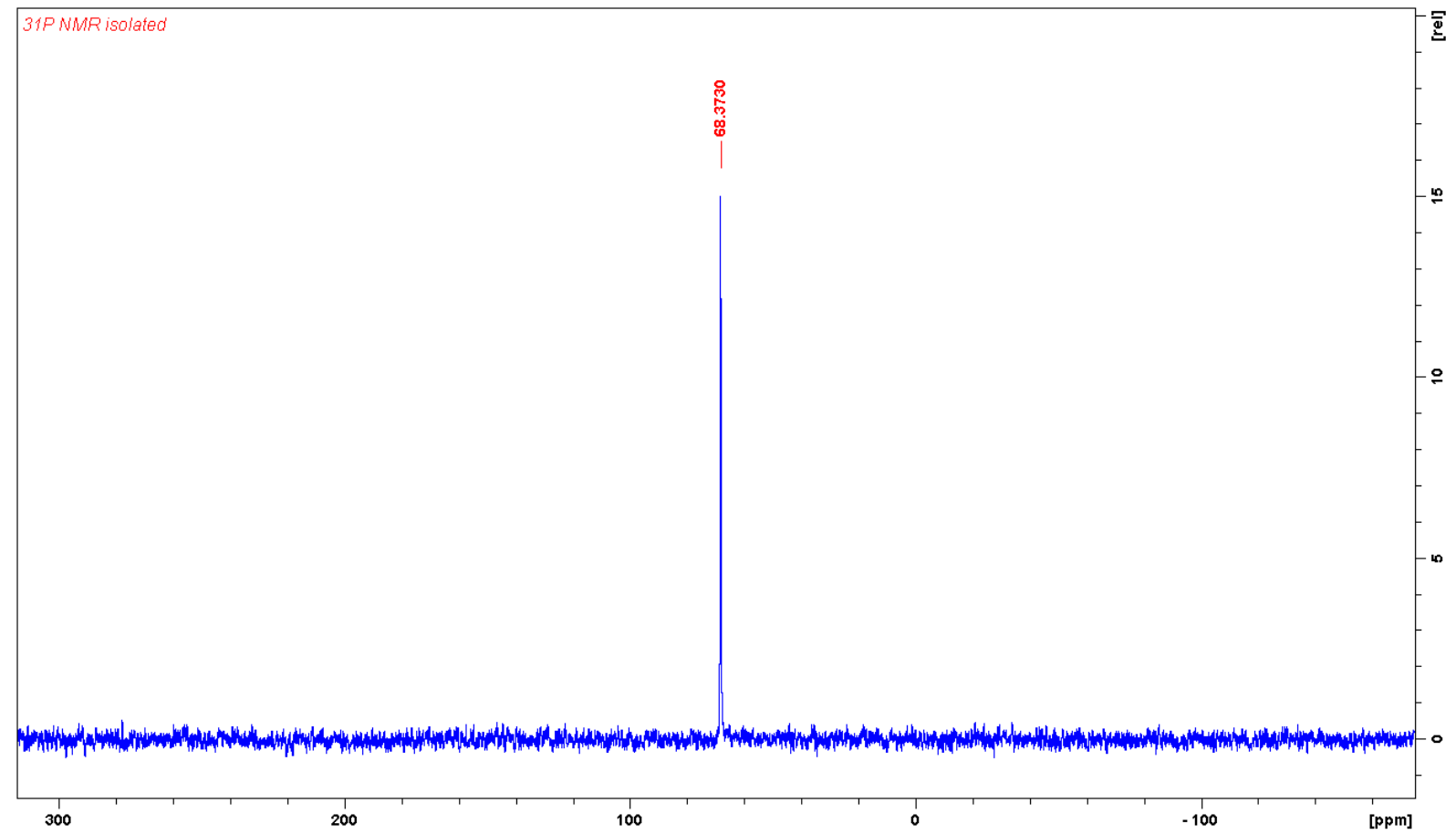

Scheme S31: ${ }^{31} \mathrm{P}$ NMR spectrum of $\left[\mathrm{Sn}\left(\mathrm{OPEt}_{3}\right)\left(\mathrm{BIMEt}_{3}\right)\right][\mathrm{OTf}]_{2}$

[Ge(OPEt3)(BIMEt3)][OTf $]_{2}$

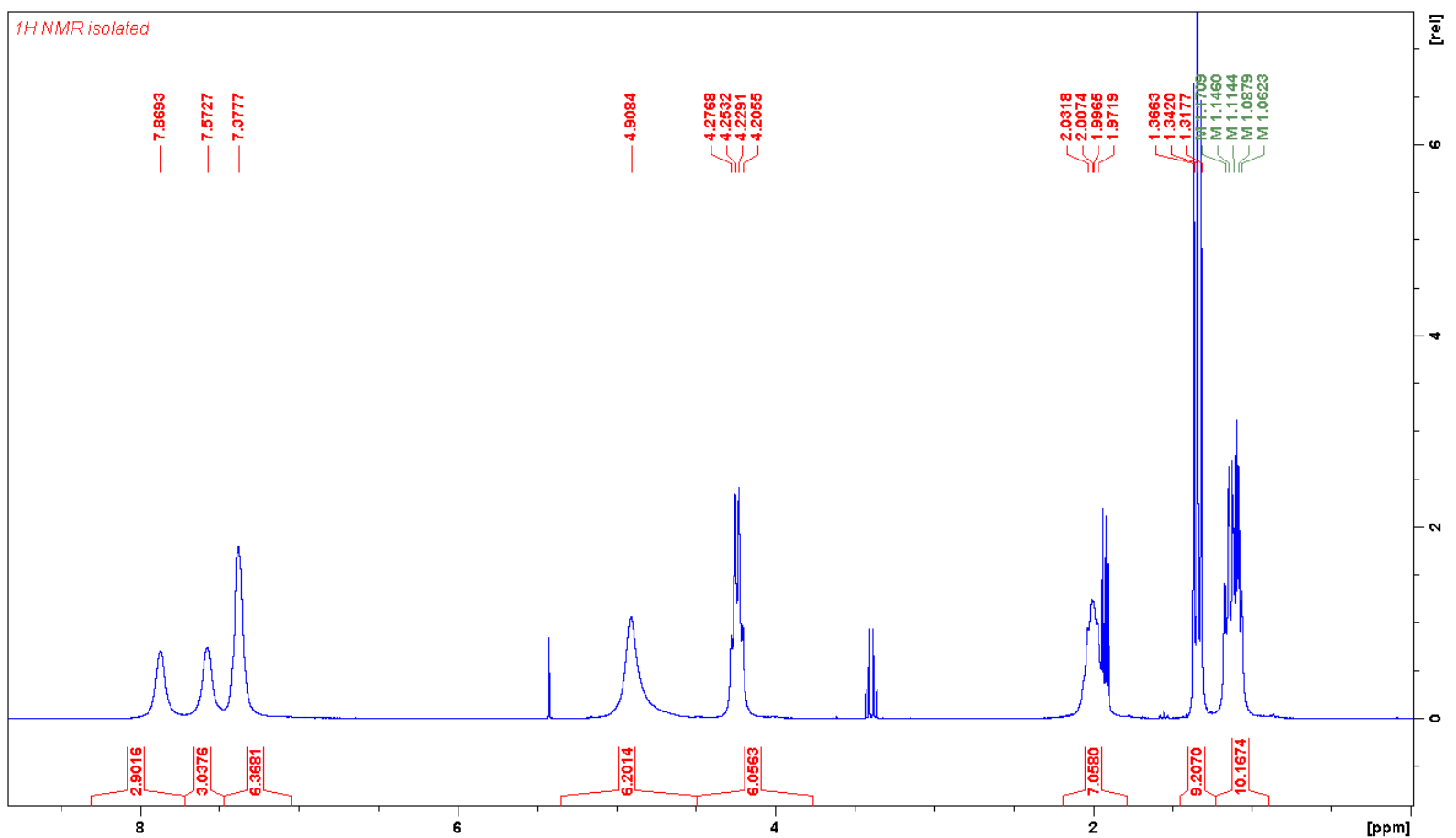

Scheme S32: ${ }^{1} \mathrm{H}$ NMR spectrum of [Ge(OPEt 3$\left.)\left(\mathrm{BIMEt}_{3}\right)\right][\mathrm{OTf}]_{2}$ with signals belonging to diethyl ether (3.4 and $1.1 \mathrm{ppm})$ and for $\mathrm{CH}_{2} \mathrm{Cl}_{2}(5.44 \mathrm{ppm})$. 


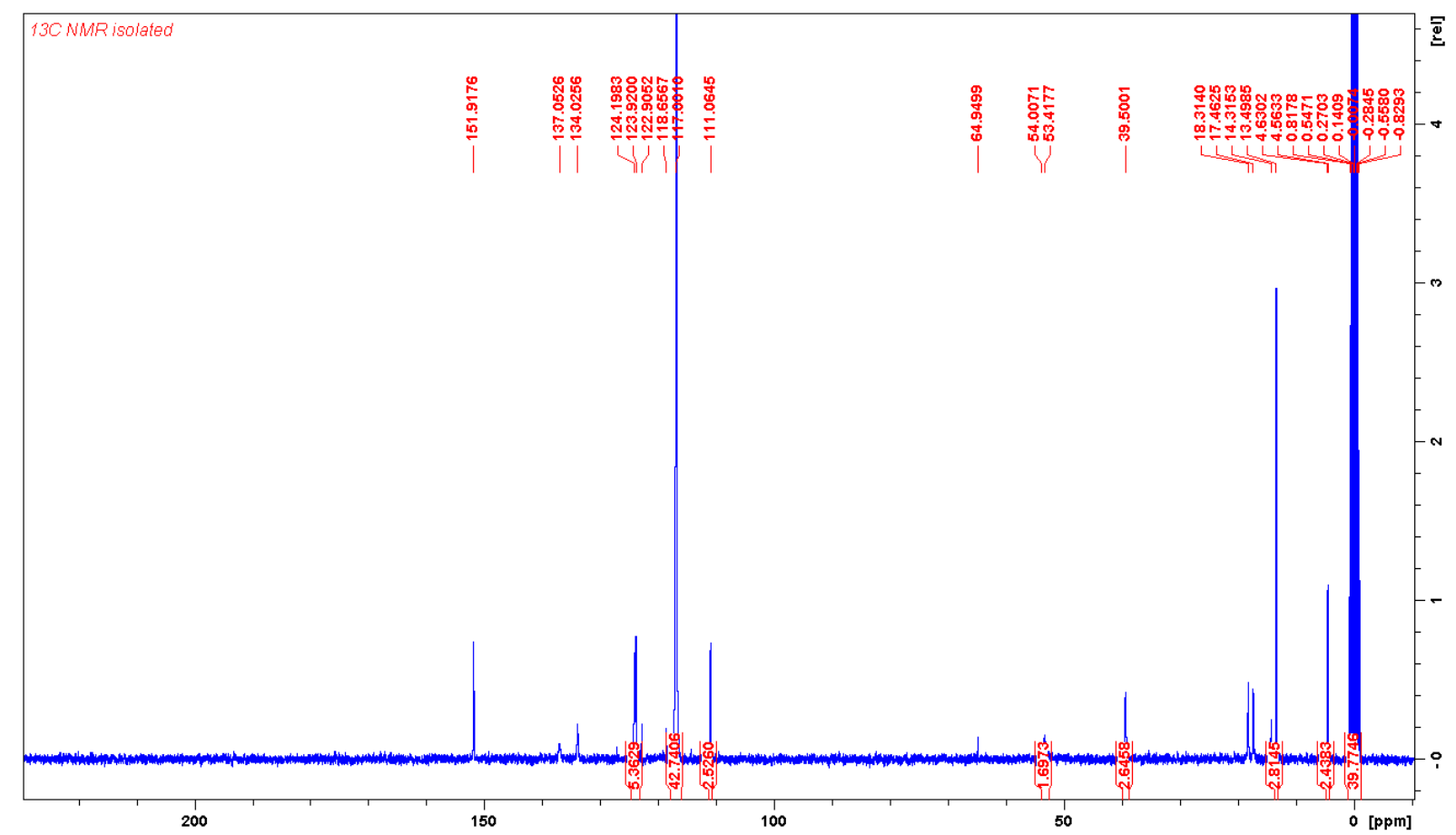

Scheme S33: ${ }^{13} \mathrm{C}$ NMR spectrum of $\left[\mathrm{Ge}\left(\mathrm{OPEt}_{3}\right)\left(\mathrm{BIMEt}_{3}\right)\right][\mathrm{OTf}]_{2}$

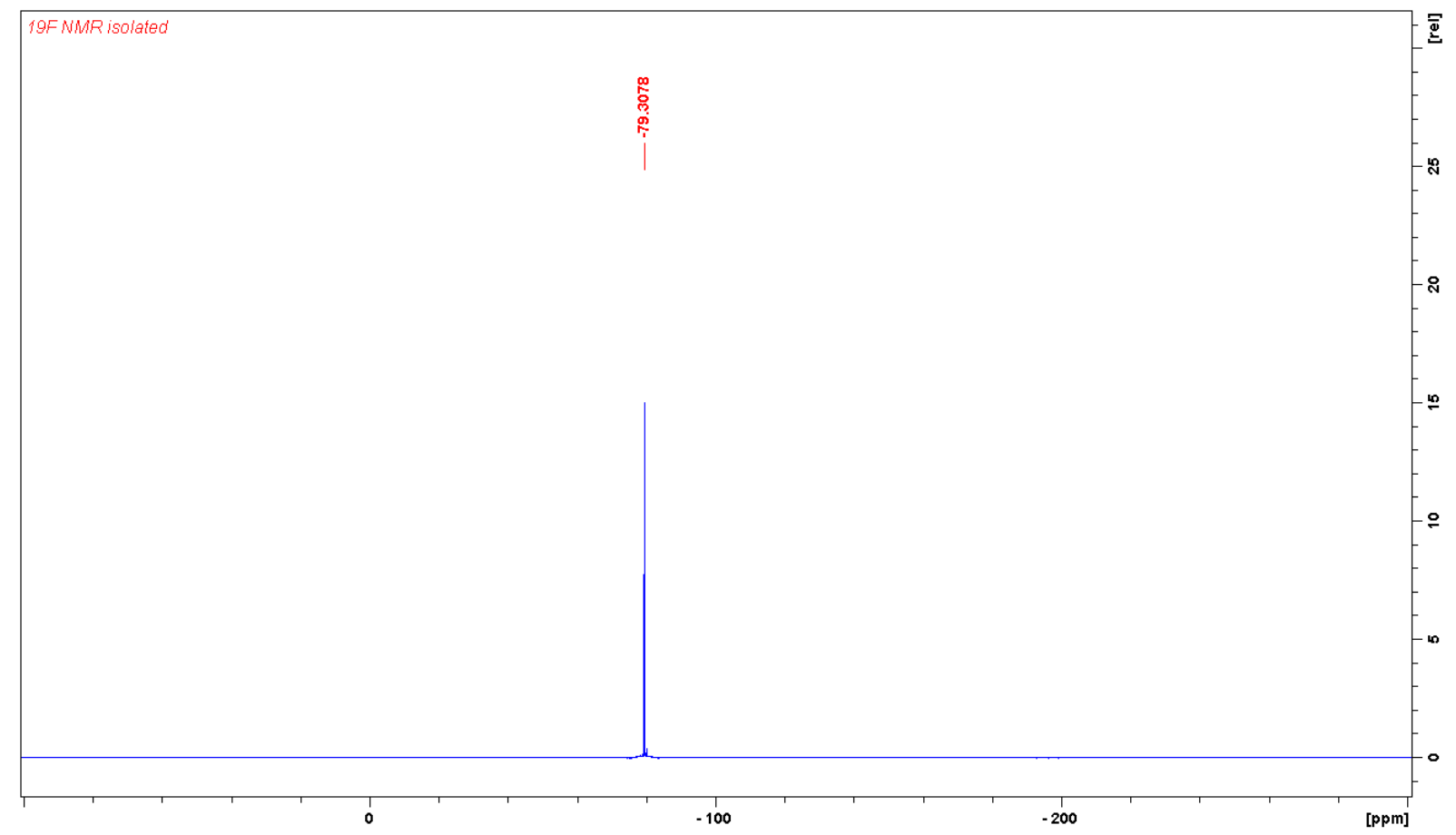

Scheme S34: ${ }^{19} \mathrm{~F}$ NMR spectrum of $\left[\mathrm{Ge}\left(\mathrm{OPEt}_{3}\right)\left(\mathrm{BIMEt}_{3}\right)\right][\mathrm{OTf}]_{2}$ 


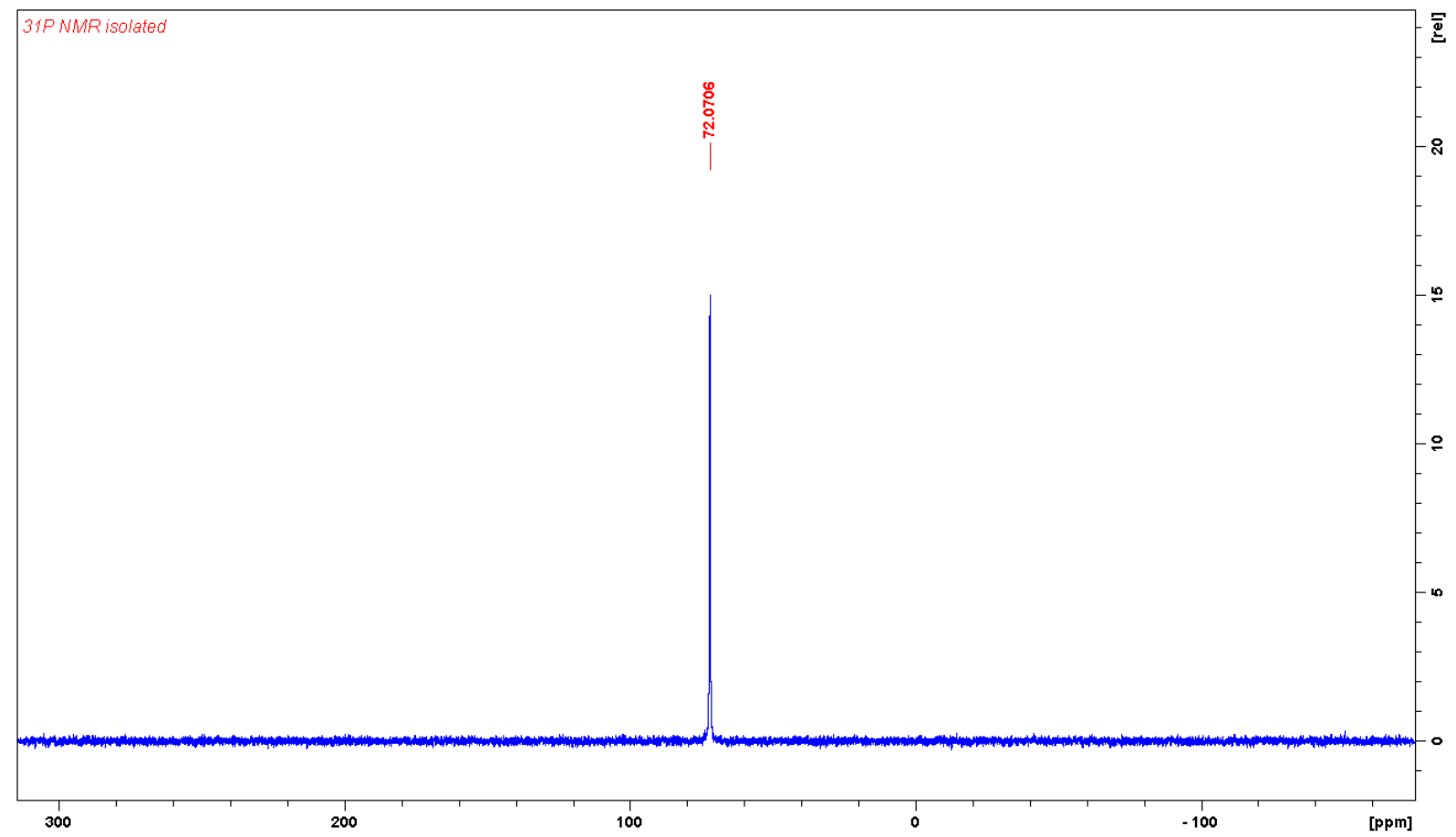

Scheme S35: ${ }^{31} \mathrm{P}$ NMR spectrum of $\left[\mathrm{Ge}\left(\mathrm{OPEt}_{3}\right)\left(\mathrm{BIMEt}_{3}\right)\right][\mathrm{OTf}]_{2}$

$\left[\left\{\operatorname{GeS}\left(\text { BIMEt}_{3}\right)\right\}_{2}\right][\text { OTf }]_{4}$

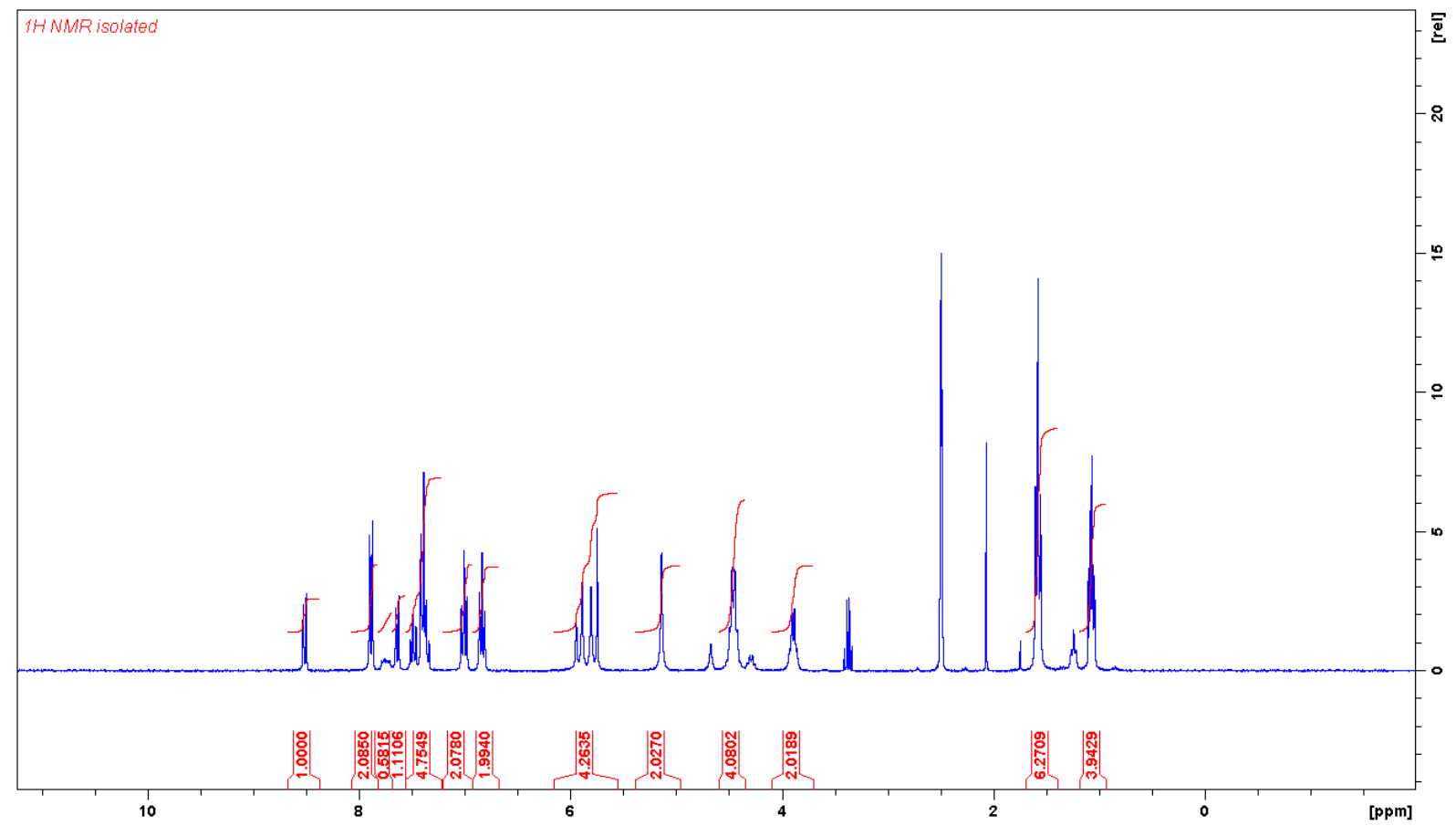

Scheme S36: ${ }^{1} \mathrm{H}$ NMR spectrum of $\left[\left\{\mathrm{GeS}\left(\mathrm{BIMEt}_{3}\right)\right\}_{2}\right][\mathrm{OTf}]_{4}$ with signals belonging to diethyl ether $(3.4$ and 1.1 ppm) and for $\mathrm{CH}_{2} \mathrm{Cl}_{2}(5.44 \mathrm{ppm})$. 


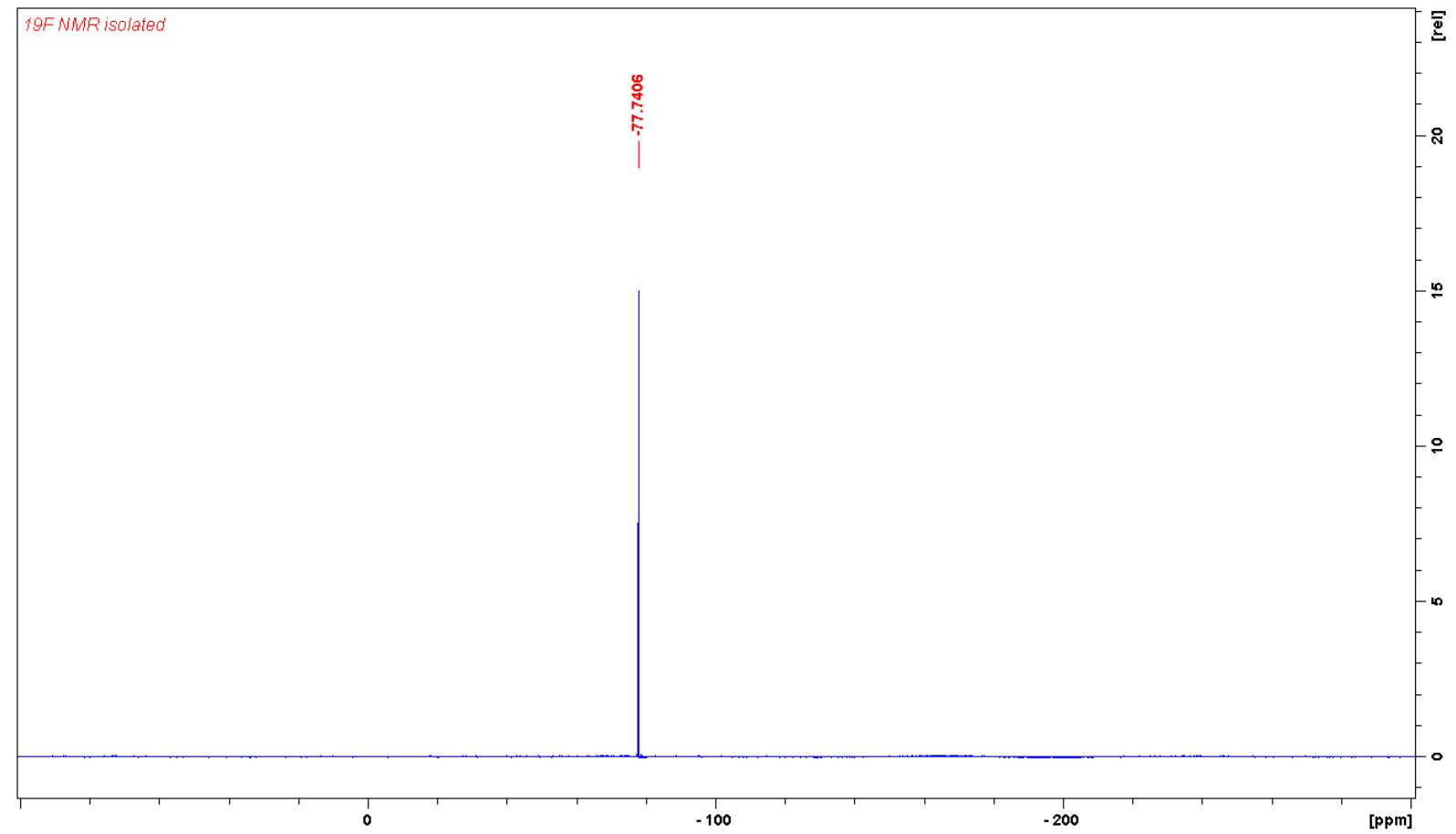

Scheme S37: ${ }^{19} \mathrm{~F}$ NMR spectrum of $\left[\left\{\mathrm{GeS}\left(\mathrm{BIMEt}_{3}\right)\right\}_{2}\right][\mathrm{OTf}]_{4}$

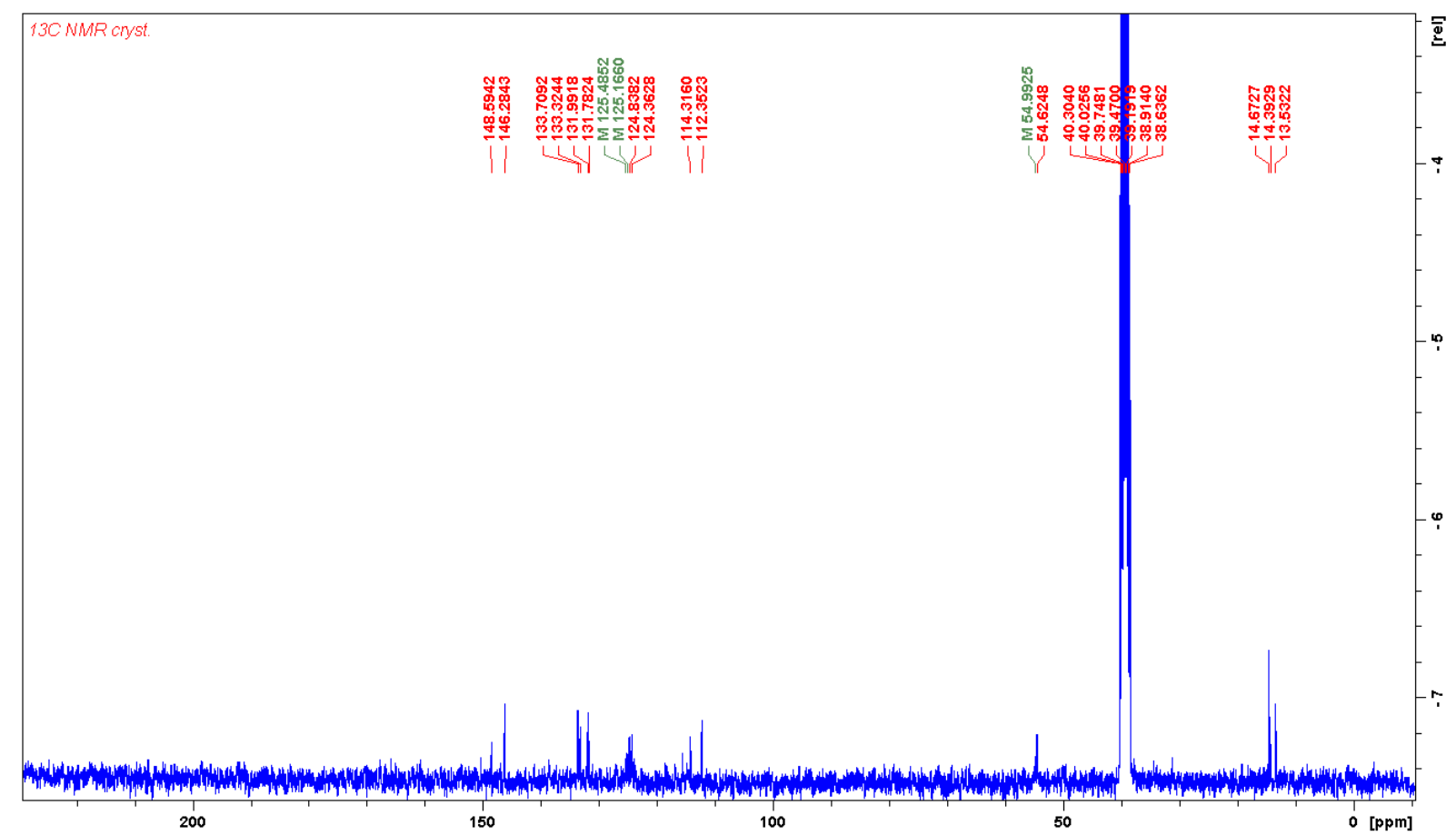

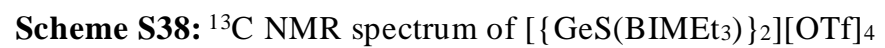




\section{X-ray Tables}

Table S1 Crystal data and structure refinement for [(BIMEt 3$\left.) \mathrm{SnBr}_{2}\right][\mathrm{OTf}]_{2}$

Identification code

CCDC

Empirical formula

Formula weight

Temperature/K

Crystal system

Space group

a/ $\AA$

$\mathrm{b} / \AA$

c/

$\alpha /{ }^{\circ}$

$\beta /{ }^{\circ}$

$\gamma /{ }^{\circ}$

Volume $/ \AA^{3}$

Z

$\rho_{\text {calc }} \mathrm{g} / \mathrm{cm}^{3}$

$\mu / \mathrm{mm}^{-1}$

$\mathrm{F}(000)$

Crystal size $/ \mathrm{mm}^{3}$

Radiation

$2 \Theta$ range for data collection/ ${ }^{\circ}$

Index ranges

Reflections collected

Independent reflections

Data/restraints/parameters

Goodness-of-fit on $\mathrm{F}^{2}$

Final $R$ indexes $[\mathrm{I}>=2 \sigma(\mathrm{I})]$

Final $\mathrm{R}$ indexes [all data]

Largest diff. peak/hole / e $\AA^{-3}$

Flack parameter
$\left[\left(\mathrm{BIMEt}_{3}\right) \mathrm{SnBr}_{2}\right][\mathrm{OTf}]_{2}$

1847487

$\mathrm{C}_{36} \mathrm{H}_{39} \mathrm{Br}_{2} \mathrm{~F}_{6} \mathrm{~N}_{9} \mathrm{O}_{6} \mathrm{~S}_{2} \mathrm{Sn}$

1150.39

170.0

triclinic

P1

11.4587(6)

12.3512(7)

$16.1116(9)$

$75.979(2)$

$88.585(2)$

$88.045(2)$

2210.7(2)

2

1.728

2.560

1144.0

$0.2 \times 0.115 \times 0.11$

$\operatorname{MoK} \alpha(\lambda=0.71073)$

5.974 to 55.022

$-14 \leq \mathrm{h} \leq 14,-16 \leq \mathrm{k} \leq 16,-20 \leq 1 \leq 20$

103404

$19297\left[\mathrm{R}_{\text {int }}=0.0583, \mathrm{R}_{\text {sigma }}=0.0384\right]$

$19297 / 3 / 1127$

1.027

$\mathrm{R}_{1}=0.0329, \mathrm{wR}_{2}=0.0695$

$\mathrm{R}_{1}=0.0448, \mathrm{wR}_{2}=0.0750$

$1.20 /-0.65$

$-0.008(2)$ 
Table S2 Crystal data and structure refinement for $\left[\left\{\left(\mathrm{BIMEt}_{3}\right)(\mathrm{GeS})\right\}_{2}\right] \mathrm{OTf}_{4}$

Identification code

CCDC

Empirical formula

Formula weight

Temperature/K

Crystal system

Space group

$\mathrm{a} / \AA$

$\mathrm{b} / \AA$

c/

$\alpha /{ }^{\circ}$

$\beta /{ }^{\circ}$

$\gamma /{ }^{\circ}$

Volume $/ \AA^{3}$

$\mathrm{Z}$

$\rho_{\mathrm{calc}} \mathrm{g} / \mathrm{cm}^{3}$

$\mu / \mathrm{mm}^{-1}$

$\mathrm{F}(000)$

Crystal size $/ \mathrm{mm}^{3}$

Radiation

$2 \Theta$ range for data collection $/^{\circ}$

Index ranges

Reflections collected

Independent reflections

Data/restraints/parameters

Goodness-of-fit on $\mathrm{F}^{2}$

Final $R$ indexes $[\mathrm{I}>=2 \sigma(\mathrm{I})]$

Final $\mathrm{R}$ indexes [all data]

Largest diff. peak/hole / e $\AA^{-3}$
$\left[\left\{\left(\mathrm{BIMEt}_{3}\right)(\mathrm{GeS})\right\}_{2}\right] \mathrm{OTf}_{4}$

1847486

$\mathrm{C}_{36} \mathrm{H}_{39} \mathrm{~F}_{6} \mathrm{GeN}_{9} \mathrm{O}_{6} \mathrm{~S}_{3}$

976.53

170.0

monoclinic

$\mathrm{P} 21 / \mathrm{c}$

$14.5688(8)$

15.8600(9)

18.1110(10)

90

90.758(2)

90

4184.4(4)

4

1.550

0.965

2000.0

$0.3 \times 0.2 \times 0.2$

$\operatorname{MoK} \alpha(\lambda=0.71073)$

5.85 to 52

$-17 \leq \mathrm{h} \leq 17,-19 \leq \mathrm{k} \leq 19,-21 \leq 1 \leq 22$

111592

$8202\left[\mathrm{R}_{\text {int }}=0.0478, \mathrm{R}_{\text {sigma }}=0.0199\right]$

$8202 / 35 / 573$

1.049

$\mathrm{R}_{1}=0.0519, \mathrm{wR}_{2}=0.1236$

$\mathrm{R}_{1}=0.0639, \mathrm{wR}_{2}=0.1342$

2.94/-1.32 
Table S3 Crystal data and structure refinement for [(BIMEt 3$) \mathrm{GeCl}_{2}$ ] $\mathrm{OTf}_{2}$

Identification code

CCDC

Empirical formula

Formula weight

Temperature/K

Crystal system

Space group

$\mathrm{a} / \AA$

$\mathrm{b} / \AA$

c/

$\alpha /{ }^{\circ}$

$\beta /{ }^{\circ}$

$\gamma /{ }^{\circ}$

Volume $/ \AA^{3}$

$\mathrm{Z}$

$\rho_{\mathrm{calc}} \mathrm{g} / \mathrm{cm}^{3}$

$\mu / \mathrm{mm}^{-1}$

$\mathrm{F}(000)$

Crystal size $/ \mathrm{mm}^{3}$

Radiation

$2 \Theta$ range for data collection ${ }^{\circ}$

Index ranges

Reflections collected

Independent reflections

Data/restraints/parameters

Goodness-of-fit on $\mathrm{F}^{2}$

Final $R$ indexes $[\mathrm{I}>=2 \sigma(\mathrm{I})]$

Final $\mathrm{R}$ indexes [all data]

Largest diff. peak/hole / e $\AA^{-3}$
[(BIMEt 3$\left.) \mathrm{GeCl}_{2}\right] \mathrm{OTf}_{2}$

1847488

$\mathrm{C}_{32} \mathrm{H}_{33} \mathrm{Cl}_{2} \mathrm{~F}_{6} \mathrm{GeN}_{7} \mathrm{O}_{6} \mathrm{~S}_{2}$

933.26

170.0

monoclinic

$\mathrm{P} 21 / \mathrm{n}$

14.2560(7)

$18.1290(8)$

$15.0748(6)$

90

103.8881(17)

90

3782.1(3)

4

1.639

1.145

1896.0

$0.205 \times 0.177 \times 0.07$

$\operatorname{MoK} \alpha(\lambda=0.71073)$

5.716 to 61.184

$-20 \leq \mathrm{h} \leq 20,-25 \leq \mathrm{k} \leq 25,-20 \leq 1 \leq 21$

169799

$11587\left[\mathrm{R}_{\text {int }}=0.0575, \mathrm{R}_{\text {sigma }}=0.0254\right]$

$11587 / 30 / 600$

1.104

$\mathrm{R}_{1}=0.0509, \mathrm{wR}_{2}=0.1110$

$\mathrm{R}_{1}=0.0853, \mathrm{wR}_{2}=0.1373$

$0.83 /-0.73$ 
Table S4 Crystal data and structure refinement for $\left(\mathrm{BIMEt}_{3}\right) \mathrm{SnI}_{2} \mathrm{OTf}_{2}$

Identification code

CCDC

Empirical formula

Formula weight

Temperature/K

Crystal system

Space group

$\mathrm{a} / \AA$

$\mathrm{b} / \AA$

c/

$\alpha /{ }^{\circ}$

$\beta /{ }^{\circ}$

$\gamma /{ }^{\circ}$

$\mathrm{Z}$

$\rho_{\mathrm{calc}} \mathrm{g} / \mathrm{cm}^{3}$

$\mu / \mathrm{mm}^{-1}$

$\mathrm{F}(000)$

Crystal size $/ \mathrm{mm}^{3}$

Radiation
Volume $/ \AA^{3}$

[(BIMEt $\left.\left.{ }_{3}\right) \mathrm{SnI}_{2}\right] \mathrm{OTf}_{2}$

1847489

$\mathrm{C}_{36} \mathrm{H}_{39} \mathrm{~F}_{6} \mathrm{I}_{2} \mathrm{~N}_{9} \mathrm{O}_{6} \mathrm{~S}_{2} \mathrm{Sn}$

1244.37

170.0

triclinic

P-1

10.9680(6)

13.1496(8)

$16.9489(10)$

99.473(2)

104.824(2)

$102.947(2)$

2238.2(2)

2

1.846

2.122

1216.0

$0.25 \times 0.075 \times 0.075$

$\operatorname{MoK} \alpha(\lambda=0.71073)$

$2 \Theta$ range for data collection ${ }^{\circ} 5.832$ to 58

Index ranges

$-14 \leq \mathrm{h} \leq 14,-17 \leq \mathrm{k} \leq 17,-23 \leq 1 \leq 23$

137927

$11862\left[\mathrm{R}_{\text {int }}=0.0441, \mathrm{R}_{\text {sigma }}=0.0203\right]$

$11862 / 0 / 549$

1.113

Goodness-of-fit on $\mathrm{F}^{2}$

$\mathrm{R}_{1}=0.0332, \mathrm{wR}_{2}=0.0700$

$\mathrm{R}_{1}=0.0498, \mathrm{wR}_{2}=0.0831$

$\begin{array}{ll}\text { Final } \mathrm{R} \text { indexes [all data] } & \mathrm{R}_{1}=0.0498, \text { Lakgest diff. peak/hole / e } \AA^{-3} \quad 2.34 /-1.18\end{array}$ 
Table S5 Crystal data and structure refinement for [(BIMEt 3$) \mathrm{Ge}(\mathrm{OPic}) \mathrm{OTf}_{2}$.

Identification code

CCDC

Empirical formula

Formula weight

Temperature/K

Crystal system

Space group

$\mathrm{a} / \AA$

$\mathrm{b} / \AA$

c/

$\alpha /{ }^{\circ}$

$\beta /{ }^{\circ}$

$\gamma /{ }^{\circ}$

Volume $/ \AA^{3}$

$\mathrm{Z}$

$\rho_{\text {calc }} \mathrm{g} / \mathrm{cm}^{3}$

$\mu / \mathrm{mm}^{-1}$

$\mathrm{F}(000)$

Crystal size $/ \mathrm{mm}^{3}$

Radiation

$2 \Theta$ range for data collection ${ }^{\circ}$

Index ranges

Reflections collected

Independent reflections

Data/restraints/parameters

Goodness-of-fit on $\mathrm{F}^{2}$

Final $R$ indexes $[\mathrm{I}>=2 \sigma(\mathrm{I})]$

Final $\mathrm{R}$ indexes [all data]

Largest diff. peak/hole / e $\AA^{-3}$
[(BIMEt $\left.{ }_{3}\right) \mathrm{Ge}(\mathrm{OPic}) \mathrm{Otf}_{2}$

1843707

$\mathrm{C}_{38} \mathrm{H}_{40} \mathrm{~F}_{6} \mathrm{GeN}_{8} \mathrm{O}_{7} \mathrm{~S}_{2}$

971.49

173.15

monoclinic

$\mathrm{P} 2{ }_{1} / \mathrm{n}$

12.1557(4)

15.9087(5)

21.9943(8)

90

$101.135(2)$

90

4173.2(2)

4

1.546

2.691

1992.0

$0.2 \times 0.17 \times 0.056$

$\mathrm{CuK} \alpha(\lambda=1.54178)$

6.902 to 145.154

$-14 \leq \mathrm{h} \leq 14,-19 \leq \mathrm{k} \leq 19,-27 \leq 1 \leq 27$

28996

$8143\left[\mathrm{R}_{\text {int }}=0.0390, \mathrm{R}_{\text {sigma }}=0.0424\right]$

$8143 / 0 / 597$

1.035

$\mathrm{R}_{1}=0.0441, \mathrm{wR}_{2}=0.1131$

$\mathrm{R}_{1}=0.0487, \mathrm{wR}_{2}=0.1174$

$0.89 /-0.62$ 
Table S6 Crystal data and structure refinement for [(BIMEt 3$) \operatorname{Sn}\left(\mathrm{OPEt}_{3}\right) \mathrm{OTf}_{2}$.

Identification code

CCDC

Empirical formula

Formula weight

Temperature/K

Crystal system

Space group

$\mathrm{a} / \AA$

$\mathrm{b} / \AA$

c/A

$\alpha /{ }^{\circ}$

$\beta /{ }^{\circ}$

$\gamma /{ }^{\circ}$

Volume $/ \AA^{3}$

$\mathrm{Z}$

$\rho_{\text {calc }} \mathrm{g} / \mathrm{cm}^{3}$

$\mu / \mathrm{mm}^{-1}$

$\mathrm{F}(000)$

Crystal size $/ \mathrm{mm}^{3}$

Radiation

$2 \Theta$ range for data collection ${ }^{\circ}$

Index ranges

Reflections collected

Independent reflections

Data/restraints/parameters

Goodness-of-fit on $\mathrm{F}^{2}$

Final $R$ indexes $[\mathrm{I}>=2 \sigma(\mathrm{I})]$

Final $\mathrm{R}$ indexes [all data]

Largest diff. peak/hole / e $\AA^{-3}$
[(BIMEt 3$) \mathrm{Sn}\left(\mathrm{OPEt}_{3}\right) \mathrm{OTf}_{2}$

1843706

$\mathrm{C}_{40} \mathrm{H}_{53} \mathrm{~F}_{6} \mathrm{~N}_{7} \mathrm{O}_{7.5} \mathrm{PS}_{2} \mathrm{Sn}$

1079.67

173.15

triclinic

P-1

13.0593(4)

$18.2861(5)$

20.0456(5)

$89.4144(14)$

83.9533(12)

88.7769(12)

4759.1(2)

4

1.507

6.096

2212.0

$0.34 \times 0.14 \times 0.126$

$\mathrm{CuK} \alpha(\lambda=1.54178)$

4.834 to 144.946

$-16 \leq \mathrm{h} \leq 16,-22 \leq \mathrm{k} \leq 22,-24 \leq 1 \leq 24$

32836

$18107\left[\mathrm{R}_{\text {int }}=0.0361, \mathrm{R}_{\text {sigma }}=0.0418\right]$

$18107 / 0 / 1168$

1.032

$\mathrm{R}_{1}=0.0477, \mathrm{wR}_{2}=0.1320$

$\mathrm{R}_{1}=0.0503, \mathrm{wR}_{2}=0.1353$

$1.44 /-1.47$ 
Table S7 Crystal data and structure refinement for [(BIMEt 3$) \operatorname{Sn}(\mathrm{OPic}) \mathrm{OTf}_{2}$.

Identification code

CCDC

Empirical formula

Formula weight

Temperature/K

Crystal system

Space group

$\mathrm{a} / \AA$

$\mathrm{b} / \AA$

c/

$\alpha /{ }^{\circ}$

$\beta /{ }^{\circ}$

$\gamma /{ }^{\circ}$

Volume $/ \AA^{3}$

$\mathrm{Z}$

$\rho_{\text {calc }} \mathrm{g} / \mathrm{cm}^{3}$

$\mu / \mathrm{mm}^{-1}$

$\mathrm{F}(000)$

Crystal size $/ \mathrm{mm}^{3}$

Radiation

$2 \Theta$ range for data collection ${ }^{\circ}$

Index ranges

Reflections collected

Independent reflections

Data/restraints/parameters

Goodness-of-fit on $\mathrm{F}^{2}$

Final $R$ indexes $[\mathrm{I}>=2 \sigma(\mathrm{I})]$

Final $\mathrm{R}$ indexes [all data]

Largest diff. peak/hole / e $\AA^{-3}$
$\left[\left(\mathrm{BIMEt}_{3}\right) \mathrm{Sn}(\mathrm{OPic})\right] \mathrm{Otf}_{2}$

1844125

$\mathrm{C}_{38} \mathrm{H}_{40} \mathrm{~F}_{6} \mathrm{~N}_{8} \mathrm{O}_{7} \mathrm{~S}_{2} \mathrm{Sn}$

1017.59

173.15

triclinic

P-1

12.9579(5)

$13.0179(5)$

$13.1613(5)$

73.687(2)

79.959(2)

$83.265(2)$

2092.61(14)

2

1.615

6.545

1032.0

$0.386 \times 0.36 \times 0.176$

$\mathrm{CuK} \alpha(\lambda=1.54178)$

6.946 to 145.544

$-16 \leq \mathrm{h} \leq 16,-16 \leq \mathrm{k} \leq 15,-16 \leq 1 \leq 16$

14613

$7984\left[\mathrm{R}_{\text {int }}=0.0329, \mathrm{R}_{\text {sigma }}=0.0420\right]$

$7984 / 36 / 629$

1.049

$\mathrm{R}_{1}=0.0544, \mathrm{wR}_{2}=0.1367$

$\mathrm{R}_{1}=0.0557, \mathrm{wR}_{2}=0.1377$

2.25/-1.98 


\section{Calculation details}

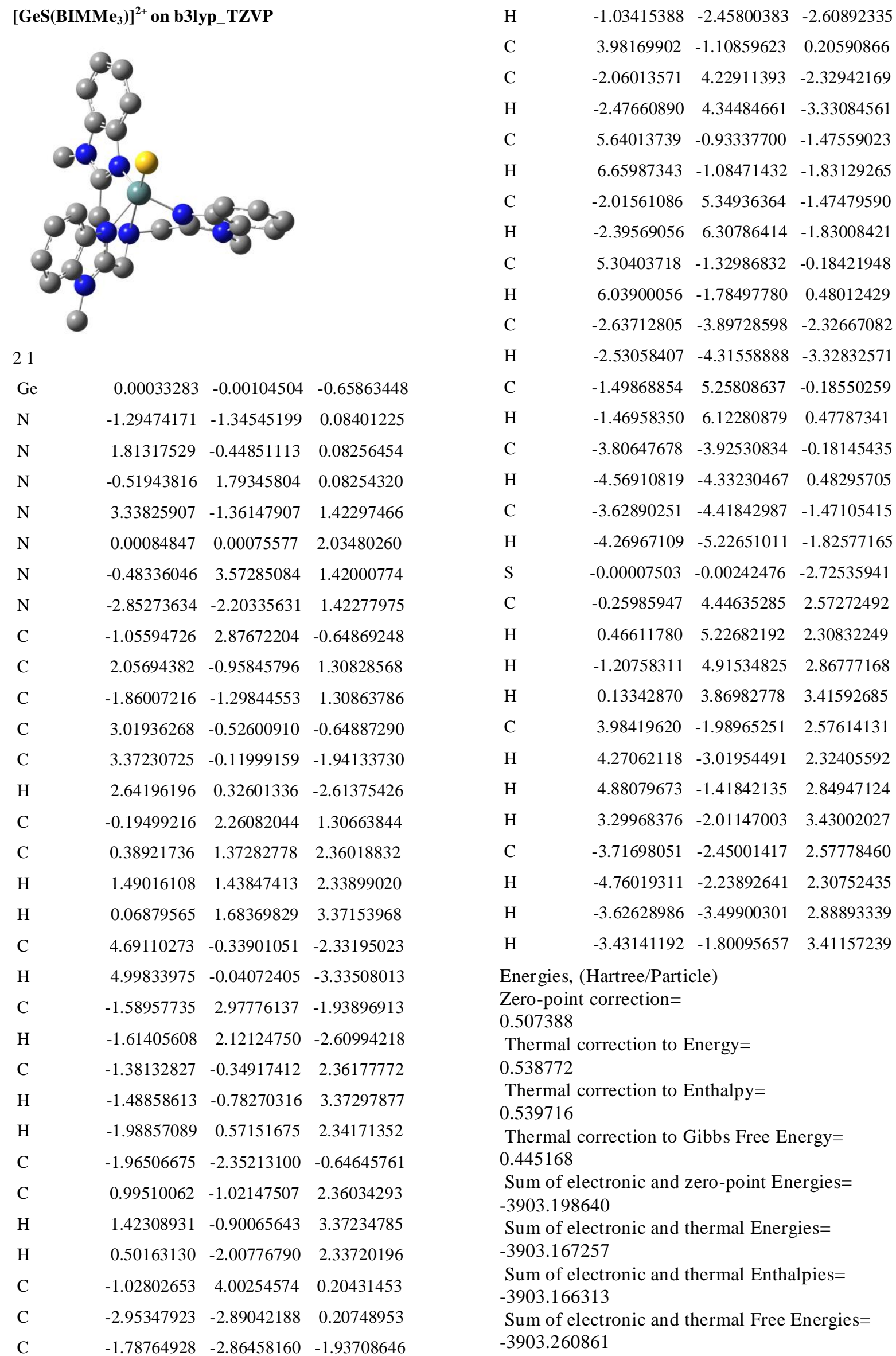




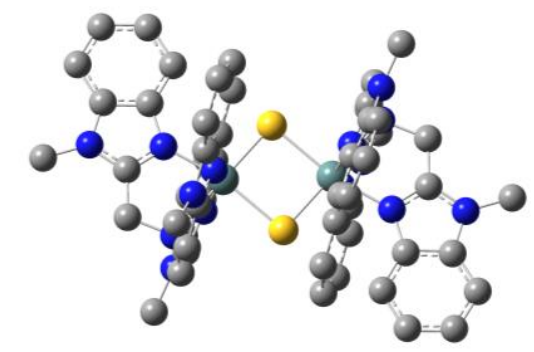

41

$\mathrm{Ge}$

$\mathrm{S}$

$\mathrm{N}$

$\mathrm{N}$

$\mathrm{N}$

$\mathrm{N}$

$\mathrm{N}$

C

C

C

C

$\mathrm{H}$

$\mathrm{H}$

C

$\mathrm{H}$

$\mathrm{H}$

$\mathrm{H}$

$\mathrm{H}$

C

C

C

$\mathrm{H}$

C

$\mathrm{H}$

C

$\mathrm{H}$

$\mathrm{C}$

$\mathrm{H}$

C

$\mathrm{H}$

C

$\mathrm{H}$

C

$\mathrm{H}$

$\mathrm{Ge}$

S

N

$\mathrm{N}$ $\begin{array}{lll}1.60500791 & -0.00014902 & 0.34069017\end{array}$

$\begin{array}{llll}0.43075086 & -0.00000563 & -1.58961620\end{array}$

$\begin{array}{lll}1.96765698 & -2.00599553 & 0.76597126\end{array}$

$\begin{array}{llll}3.52273993 & -0.00034631 & -0.35166662\end{array}$

$\begin{array}{lll}1.96812731 & 2.00557818 & 0.76600939\end{array}$

$\begin{array}{lll}5.71008705 & -0.00039337 & -0.00348268\end{array}$

$\begin{array}{lll}2.77191780 & -0.00034722 & 2.35450137\end{array}$

$\begin{array}{llll}2.51405575 & 3.63342216 & 2.17059218\end{array}$

$\begin{array}{lll}2.51325051 & -3.63401536 & 2.17048117\end{array}$

$\begin{array}{llll}2.02505533 & 3.18871836 & 0.02340423\end{array}$

$\begin{array}{lll}4.49225864 & -0.00041550 & 0.55909971\end{array}$

$\begin{array}{lll}2.27522074 & -2.31928303 & 2.02130603\end{array}$

$\begin{array}{lll}4.14403846 & -0.00030261 & -1.61233622\end{array}$

$3.64763478-0.00029831-2.91652890$

$\begin{array}{lll}2.58811648 & -0.00030305 & -3.12276468\end{array}$

$\begin{array}{lll}2.27581052 & 2.31873485 & 2.02134572\end{array}$

$\begin{array}{lll}2.35736095 & 1.24333676 & 3.05805651\end{array}$

$\begin{array}{lll}3.05049895 & 1.49599861 & 3.86272302\end{array}$

$\begin{array}{lll}1.37447605 & 1.07633591 & 3.50235970\end{array}$

$\begin{array}{lll}4.57064968 & -0.00029835 & -3.95009182\end{array}$

$\begin{array}{llll}4.21843133 & -0.00028559 & -4.97329702\end{array}$

$\begin{array}{lll}1.86324901 & 3.45371793 & -1.33713513\end{array}$

$\begin{array}{llll}1.62439600 & 2.66807785 & -2.03823813\end{array}$

$\begin{array}{lll}2.35692774 & -1.24391475 & 3.05803251\end{array}$

$\begin{array}{llll}3.04988915 & -1.49673551 & 3.86280370\end{array}$

$\begin{array}{lll}1.37402358 & -1.07662804 & 3.50217819\end{array}$

$\begin{array}{llll}2.02442066 & -3.18910399 & 0.02329618\end{array}$

$\begin{array}{lll}4.23443138 & -0.00062887 & 2.03677951\end{array}$

$\begin{array}{lll}4.70441638 & 0.87372495 & 2.49197556\end{array}$

$\begin{array}{lll}4.70395497 & -0.87544826 & 2.49156517\end{array}$

$\begin{array}{llll}2.36232557 & 4.22310221 & 0.91423641\end{array}$

$\begin{array}{lll}2.36145042 & -4.22359737 & 0.91409100\end{array}$

$\begin{array}{lll}1.86259661 & -3.45401632 & -1.33726044\end{array}$

$\begin{array}{lll}1.62390439 & -2.66829983 & -2.03833057\end{array}$

$\begin{array}{lll}5.53061833 & -0.00032520 & -1.38789792\end{array}$

$\begin{array}{llll}2.04135155 & 4.76341819 & -1.75280916\end{array}$

$\begin{array}{lll}1.94607949 & 5.00346619 & -2.80392695\end{array}$

$\begin{array}{lll}5.95492858 & -0.00031267 & -3.71102873\end{array}$

$\begin{array}{lll}6.63795470 & -0.00031342 & -4.55009474\end{array}$

$\begin{array}{lll}2.36683369 & 5.79225632 & -0.85136635\end{array}$

$\begin{array}{llll}2.50440564 & 6.79881495 & -1.22386101\end{array}$

$\begin{array}{llll}6.46132094 & -0.00033380 & -2.42266948\end{array}$

$\begin{array}{lll}7.52752806 & -0.00034239 & -2.24005154\end{array}$

$\begin{array}{llll}2.04045238 & -4.76372962 & -1.75298612\end{array}$

$\begin{array}{llll}1.94515022 & -5.00372216 & -2.80411297\end{array}$

$\begin{array}{lll}2.53321253 & 5.54159321 & 0.50020154\end{array}$

$\begin{array}{llll}2.79759744 & 6.33369000 & 1.18793787\end{array}$

$\begin{array}{lll}2.53207630 & -5.54210942 & 0.50000625\end{array}$

$\begin{array}{llll}2.79626247 & -6.33429072 & 1.18772234\end{array}$

$\begin{array}{llll}2.36570393 & -5.79267675 & -0.85157673\end{array}$

$\begin{array}{llll}2.50310333 & -6.79924125 & -1.22412208\end{array}$

$\begin{array}{lll}-1.60500702 & 0.00015516 & -0.34069062\end{array}$

$\begin{array}{lll}-0.43075019 & 0.00001638 & 1.58961578\end{array}$

$\begin{array}{lll}-1.96766411 & 2.00599981 & -0.76597408\end{array}$

$\begin{array}{lll}-3.52273898 & 0.00034548 & 0.35166647\end{array}$ $\begin{array}{lll}-1.96811916 & -2.00557347 & -0.76600786\end{array}$

$\begin{array}{lll}-5.71008621 & 0.00038332 & 0.00348302\end{array}$

$\begin{array}{lll}-2.77191762 & 0.00034653 & -2.35450195\end{array}$

$\begin{array}{lll}-2.51404048 & -3.63342190 & -2.17058820\end{array}$

$\begin{array}{llll}-2.51326481 & 3.63401595 & -2.17048562\end{array}$

$\begin{array}{lll}-2.02504254 & -3.18871268 & -0.02340078\end{array}$

$\begin{array}{lll}-4.49225793 & 0.00040832 & -0.55909974\end{array}$

$\begin{array}{llll}-2.27523008 & 2.31928469 & -2.02130897\end{array}$

$\begin{array}{lll}-4.14403716 & 0.00029668 & 1.61233615\end{array}$

$\begin{array}{lll}-3.64763302 & 0.00029254 & 2.91652866\end{array}$

$\begin{array}{lll}-2.58811464 & 0.00030145 & 3.12276408\end{array}$

$\begin{array}{lll}-2.27580165 & -2.31873320 & -2.02134364\end{array}$

$\begin{array}{llll}-2.35735667 & -1.24333684 & -3.05805583\end{array}$

$\begin{array}{llll}-3.05049404 & -1.49600223 & -3.86272179\end{array}$

$\begin{array}{llll}-1.37447261 & -1.07633276 & -3.50235963\end{array}$

$\begin{array}{lll}-4.57064759 & 0.00028778 & 3.95009186\end{array}$

$\begin{array}{lll}-4.21842889 & 0.00027576 & 4.97329693\end{array}$

$\begin{array}{lll}-1.86323460 & -3.45370960 & 1.33713894\end{array}$

$\begin{array}{lll}-1.62438470 & -2.66806743 & 2.03824066\end{array}$

$\begin{array}{lll}-2.35693220 & 1.24391486 & -3.05803430\end{array}$

$\begin{array}{llll}-3.04989393 & 1.49673195 & -3.86280637\end{array}$

$\begin{array}{llll}-1.37402695 & 1.07663166 & -3.50217887\end{array}$

$\begin{array}{llll}-2.02443678 & 3.18910813 & -0.02329952\end{array}$

$\begin{array}{lll}-4.23443106 & 0.00062328 & -2.03677964\end{array}$

$\begin{array}{llll}-4.70441364 & -0.87373145 & -2.49197637\end{array}$

$\begin{array}{llll}-4.70395734 & 0.87544182 & -2.49156422\end{array}$

$-2.36230758 \quad-4.22309940 \quad-0.91423155$

$\begin{array}{llll}-2.36147093 & 4.22359927 & -0.91409528\end{array}$

$\begin{array}{lll}-1.86261716 & 3.45402199 & 1.33725734\end{array}$

$\begin{array}{lll}-1.62392198 & 2.66830710 & 2.03832824\end{array}$

$\begin{array}{lll}-5.53061706 & 0.00031414 & 1.38789827\end{array}$

$\begin{array}{lll}-2.04133010 & -4.76341021 & 1.75281468\end{array}$

$\begin{array}{lll}-1.94605618 & -5.00345648 & 2.80393268\end{array}$

$\begin{array}{lll}-5.95492657 & 0.00029695 & 3.71102919\end{array}$

$\begin{array}{lll}-6.63795242 & 0.00029412 & 4.55009542\end{array}$

$\begin{array}{lll}-2.36680698 & -5.79225129 & 0.85137322\end{array}$

$\begin{array}{lll}-2.50437317 & -6.79881019 & 1.22386923\end{array}$

$\begin{array}{lll}-6.46131935 & 0.00031751 & 2.42267007\end{array}$

$\begin{array}{lll}-7.52752652 & 0.00032185 & 2.24005242\end{array}$

$\begin{array}{lll}-2.04048144 & 4.76373446 & 1.75298212\end{array}$

$\begin{array}{lll}-1.94518291 & 5.00372791 & 2.80410910\end{array}$

$\begin{array}{lll}-2.53318746 & -5.54159082 & -0.50019493\end{array}$

$\begin{array}{llll}-2.79756779 & -6.33369000 & -1.18793026\end{array}$

$\begin{array}{lll}-2.53210502 & 5.54211048 & -0.50001153\end{array}$

$\begin{array}{llll}-2.79629443 & 6.33428998 & -1.18772849\end{array}$

$\begin{array}{lll}-2.36573680 & 5.79267940 & 0.85157168\end{array}$

$\begin{array}{lll}-2.50314256 & 6.79924332 & 1.22411629\end{array}$

$\begin{array}{lll}-7.01813844 & 0.00037823 & -0.66088286\end{array}$

$\begin{array}{lll}-7.57573362 & -0.88932041 & -0.37057503\end{array}$

$\begin{array}{lll}-7.57594852 & 0.88979273 & -0.37010345\end{array}$

$\begin{array}{lll}-6.89284218 & 0.00072171 & -1.74030977\end{array}$

$\begin{array}{lll}-2.87000174 & 4.36533265 & -3.39097448\end{array}$

$\begin{array}{llll}-3.83522653 & 4.85275350 & -3.25885885\end{array}$

$\begin{array}{llll}-2.11039896 & 5.11696514 & -3.60159902\end{array}$

$\begin{array}{llll}-2.92613501 & 3.68317083 & -4.23453721\end{array}$

$\begin{array}{lll}2.87107080 & 4.36459609 & 3.39108679\end{array}$

$\begin{array}{llll}3.83672268 & 4.85123580 & 3.25919502\end{array}$

$\begin{array}{lll}2.11201210 & 5.11686722 & 3.60138833\end{array}$

$\begin{array}{llll}2.92636110 & 3.68250480 & 4.23476097\end{array}$

$\begin{array}{lll}7.01813892 & -0.00039375 & 0.66088388\end{array}$

$\begin{array}{lll}7.57594190 & -0.88981518 & 0.37011199\end{array}$

$\begin{array}{lll}7.57574166 & 0.88929792 & 0.37056925\end{array}$

$\begin{array}{lll}6.89284207 & -0.00072681 & 1.74031075\end{array}$

$\begin{array}{lll}2.86998664 & -4.36533438 & 3.39096886\end{array}$

$\begin{array}{lll}2.11038180 & -5.11696490 & 3.60159296\end{array}$

$\begin{array}{lll}3.83520997 & -4.85275788 & 3.25885219\end{array}$

$2.92612216-3.68317365 \quad 4.23453235$

$\begin{array}{lll}-2.87104946 & -4.36459985 & -3.39108218\end{array}$

$\begin{array}{llll}-2.11198355 & -5.11686351 & -3.60138459\end{array}$ 
$\mathrm{H} \quad \begin{array}{llll}-3.83669629 & -4.85124907 & -3.25918875\end{array}$

$\mathrm{H} \quad \begin{array}{llll}-2.92634812 & -3.68250936 & -4.23475647\end{array}$

Energies, (Hartree/Particle)

Zero-point correction $=$

1.016071

Thermal correction to Energy=

1.080566

Thermal correction to Enthalpy=

1.081511

Thermal correction to Gibbs Free Energy=

0.915212

Sum of electronic and zero-point Energies=

$-7806.213591$

Sum of electronic and thermal Energies= $-7806.149096$

Sum of electronic and thermal Enthalpies=

$-7806.148152$

Sum of electronic and thermal Free Energies= $-7806.314450$ 